\title{
Consistent description of kinetics and hydrodynamics of weakly nonequilibrium processes in simple liquids
}

\author{
B. Markiv, I. Omelyan, M. Tokarchuk \\ Institute for Condensed Matter Physics of the National Academy of Sciences of Ukraine, \\ 1 Svientsitskii Srt., 79011 Lviv, Ukraine
}

\begin{abstract}
The generalized transport equations for a consistent description of kinetic and hydrodynamic processes in dense gases and liquids are considered. The inner structure of the generalized transport kernels for these equations is established. It is shown how in this approach to obtain the transport equation of molecular hydrodynamics. For the model potential of interaction presented as a sum of the hard spheres potential and certain long-range potential a spectrum of collective modes in the system is investigated.
\end{abstract}

PACS numbers: 05.20.Dd, 05.60.+w, 52.25.Fi, 82.20.M 


\section{INTRODUCTION}

A number of investigations [1-28] was devoted to the problem of constructing a consistent description of kinetic and hydrodynamic processes in dense gases, liquids, and plasma.

In dense gases and liquids (simple and molecular) as well as in dense plasma there is no small parameter and the characteristic time of interparticle correlations is comparable with those for the one-particle distribution function. This means that during the particles collision process the many-particles correlations related to local mass, momentum and energy conservation laws, underlying the hydrodynamic description of a system, can not be neglected. In this connection the local conservation laws impose some restrictions on the kinetic processes. Their role is especially important at high densities, when the interaction between a separate group of particles and other ones can not be neglected. This indicates a close connection between the kinetic and hydrodynamic processes in dense gases, liquids and plasma. Such peculiarities of transport processes should be displayed in the behaviour of collective excitations spectrum, time correlation functions (in particular, the dynamic structure factor) as well as the generalized transport coefficient in the region of intermediate values of wave vector $\vec{k}$ and frequency $\omega$. Another important issue remains for investigation when the nonequilibrium processes are considered during a long time (small $\omega$ ) at a small spatial scale (large $\vec{k}$ ) between particles and when the features of interparticle interaction character are manifested. In particular, it is important to note the results of comparison of experimental data on neutron scattering and molecular dynamics ones [29]. For all values of $\vec{k}$ in the region of small $\omega$, deviations are observed unlike for the region of high frequencies (short time of observation). Whereas at small $\vec{k}$ the behaviour can be explained by collective effects, obviously, the dynamics of particles scattering with a momentum exchange should be taken into account at large $\vec{k}$. This is very important from the point of view of experimental investigations on neutron scattering [1, 6, 30].

For instance, the importance of taking into account the kinetic processes connected with irreversible collision processes at the scale of short-ranged interparticle interactions was pointed out in [31]. The short-wavelength collective modes in liquids were investigated therein on the basis of the linearized kinetic equation of the revised Enskog theory for the hard spheres model.

In this paper we investigate a spectrum of collective excitations within a consistent de- 
scription of kinetic and hydrodynamic processes in a system when potential of interaction between particles consists of two parts: the hard spheres potential and a long-range part. In the second section we present basic transport equations of a consistent description of kinetics and hydrodynamics of weakly nonequilibrium processes obtained by means of the Zubarev nonequilibrium statistical operator (NSO) method [22]. The memory functions entering these equations are calculated in section 3. In the fourth section based on the transport equations of a consistent description of kinetics and hydrodynamics we obtain the equations of molecular hydrodynamics. In section 5 the latter are used for investigation of spectrum of collective excitations in the system with potential of interaction modelled by a sum of the potential of hard spheres and a certain long-range potential.

\section{KINETIC EQUATIONS FOR WEAKLY NONEQUILIBRIUM STATES}

Using the ideas of papers [11, 12] the nonequilibrium statistical operator for a consistent description of kinetic and hydrodynamic processes for a system of classical interacting particles was obtained in [20, 21] by means of the NSO method. For this purpose the following approximation for quasiequilibrium distribution function being the functional of the reduced-description parameters $\left\langle\hat{n}_{\vec{k}}(\vec{p})\right\rangle^{t}$ and $\left\langle\hat{h}_{\vec{k}}^{i n t}\right\rangle^{t}$, was used:

$$
\varrho_{q}\left(x^{N} ; t\right)=\varrho_{0}\left(x^{N}\right)\left\{1+\sum_{\vec{k}}^{\prime}\left\langle\hat{h}_{\vec{k}}^{i n t}\right\rangle^{t} \Phi_{h h}^{-1}(\vec{k}) \hat{h}_{\vec{k}}^{i n t}+\sum_{\vec{k}}^{\prime} \int d \vec{p} \int d \vec{p}^{\prime}\left\langle\hat{n}_{\vec{k}}(\vec{p})\right\rangle^{t} \Phi_{\vec{k}}^{-1}\left(\vec{p}, \vec{p}^{\prime}\right) \hat{n}_{\vec{k}}\left(\vec{p}^{\prime}\right)\right\} .
$$

Here, $\varrho_{0}\left(x^{N}\right)$ is an equilibrium distribution function, $\sum_{\vec{k}}^{\prime}=\sum_{\vec{k}(\vec{k} \neq 0)}$, with $\vec{k}$ being a wave vector.

$$
\hat{n}_{\vec{k}}(\vec{p})=\int d \vec{r} e^{-i \vec{k} \cdot \vec{r}} \hat{n}_{1}(\vec{r}, \vec{p})
$$

are the Fourier-components of microscopic phase density of particles number,

$$
\hat{h}_{\vec{k}}^{i n t}=\hat{\varepsilon}_{\vec{k}}^{i n t}-\left\langle\hat{\varepsilon}_{\vec{k}}^{i n t} \hat{n}_{-\vec{k}}\right\rangle_{0} S_{2}^{-1}(k) \hat{n}_{\vec{k}}
$$

are the Fourier-components of the potential part of the enthalpy density, $\hat{\varepsilon}_{\vec{k}}^{\text {int }}=$ $\frac{1}{2} \sum_{l \neq j=1}^{N} \Phi\left(\left|\vec{r}_{l j}\right|\right) e^{-i \vec{k} \cdot \vec{r}_{j}}$ and $\hat{n}_{\vec{k}}=\sum_{l=1}^{N} e^{-i \vec{k} \cdot \vec{r}_{l}}$ are the Fourier-components of the potential energy and particles number densities, respectively. $\Phi_{h h}^{-1}(\vec{k})$ is the function inverse 
to the equilibrium correlation function $\Phi_{h h}(\vec{k})=\left\langle\hat{h}_{\vec{k}}^{\text {int }} \hat{h}_{-\vec{k}}^{\text {int }}\right\rangle_{0},\langle\ldots\rangle_{0}=\int d \Gamma_{N} \ldots \varrho_{0}\left(x^{N}\right)$, $\left\langle\hat{n}_{\vec{k}}(\vec{p})\right\rangle_{0}=0(\vec{k} \neq 0) . \Phi_{\vec{k}}^{-1}\left(\vec{p}, \vec{p}^{\prime}\right)$ is the function inverse to

$$
\Phi_{\vec{k}}\left(\vec{p}, \vec{p}^{\prime}\right)=\left\langle\hat{n}_{\vec{k}}(\vec{p}) \hat{n}_{-\vec{k}}\left(\vec{p}^{\prime}\right)\right\rangle_{0}=n \delta\left(\vec{p}-\vec{p}^{\prime}\right) f_{0}\left(p^{\prime}\right)+n^{2} f_{0}(p) f_{0}\left(p^{\prime}\right) h_{2}(\vec{k}) .
$$

It equals to

$$
\Phi_{\vec{k}}^{-1}\left(\vec{p}, \vec{p}^{\prime}\right)=\frac{\delta\left(\vec{p}-\vec{p}^{\prime}\right)}{n f_{0}\left(p^{\prime}\right)}-c_{2}(k),
$$

where $n=N / V, f_{0}(p)=(\beta / 2 \pi m)^{3 / 2} \exp \left(-\beta p^{2} / 2 m\right)$ is the Maxwellian distribution, $\beta=$ $1 / k_{B} T$ with $T$ being an equilibrium value of temperature. $c_{2}(k)$ is the direct correlation function related to correlation function $h_{2}(k): h_{2}(k)=c_{2}(k)\left[1-n c_{2}(k)\right]^{-1} . S_{2}(k)=\left\langle\hat{n}_{\vec{k}} \hat{n}_{-\vec{k}}\right\rangle_{0}$ is the static structure factor. It is important to note that dynamic variables $\hat{h}_{\vec{k}}^{\text {int }}$ and $\hat{n}_{\vec{k}}(\vec{p})$ in the distribution (2.1) are orthogonal in the sense that $\left\langle\hat{h}_{\vec{k}}^{i n t} \hat{n}_{\vec{k}}(\vec{p})\right\rangle_{0}=0$.

In the approximation (2.1), within the framework of the Zubarev NSO method [22, 32], the nonequilibrium distribution function $\varrho\left(x^{N} ; t\right)$ has the following form [20, 21]:

$$
\begin{aligned}
& \varrho\left(x^{N} ; t\right)=\varrho_{0}\left(x^{N}\right)\left\{1+\sum_{\vec{k}}^{\prime} \int d \vec{p} \int d \vec{p}^{\prime}\left\langle\hat{n}_{\vec{k}}(\vec{p})\right\rangle^{t} \Phi_{\vec{k}}^{-1}\left(\vec{p}, \vec{p}^{\prime}\right) \hat{n}_{-\vec{k}}\left(\vec{p}^{\prime}\right)\right. \\
& \quad+\sum_{\vec{k}}^{\prime}\left\langle\hat{h}_{\vec{k}}^{i n t}\right\rangle^{t} \Phi_{h h}^{-1}(\vec{k}) \hat{h}_{-\vec{k}}^{i n t}-\sum_{\vec{k}}^{\prime} \int d \vec{p} \int d \vec{p}^{\prime} \int_{-\infty}^{t} e^{\varepsilon\left(t^{\prime}-t\right)} T_{0}\left(t, t^{\prime}\right) I_{n}(-\vec{k} ; \vec{p}) \Phi_{\vec{k}}^{-1}\left(\vec{p}, \vec{p}^{\prime}\right)\left\langle\hat{n}_{\vec{k}}\left(\vec{p}^{\prime}\right)\right\rangle^{t^{\prime}} \\
& \left.\quad-\sum_{\vec{k}}^{\prime} \int_{-\infty}^{t} e^{\varepsilon\left(t^{\prime}-t\right)} T_{0}\left(t, t^{\prime}\right) I_{h}^{i n t}(-\vec{k}) \Phi_{h h}^{-1}(\vec{k})\left\langle\hat{h}_{\vec{k}}^{i n t}\right\rangle^{\prime}\right\},
\end{aligned}
$$

where

$$
I_{n}(\vec{k} ; \vec{p})=\left(1-P_{0}\right) i L_{N} \hat{n}_{\vec{k}}(\vec{p})=\left(1-P_{0}\right) \dot{\hat{n}}_{\vec{k}}(\vec{p}), \quad I_{h}^{i n t}(\vec{k})=\left(1-P_{0}\right) i L_{N} \hat{h}_{\vec{k}}^{\text {int }}=\left(1-P_{0}\right) \dot{\hat{h}}_{\vec{k}}^{\text {int }}
$$

are the generalized flows in linear approximation $\left(i L_{N}\right.$ is the Liouville operator of a simple liquid). $T_{0}\left(t, t^{\prime}\right)=\exp \left[\left(t-t^{\prime}\right)\left(1-P_{0}\right) i L_{N}\right]$ is the evolution operator with regard to projection operator $P_{0}$ being a linear approximation of the Mori projection operator constructed on the orthogonal dynamic variables $\hat{n}_{\vec{k}}(\vec{p}), \hat{h}_{\vec{k}}^{\text {int }}$ [21]:

$$
P_{0} \hat{A}_{\vec{k}}=\sum_{\vec{k}}^{\prime}\left\langle\hat{A}_{\vec{k}} \hat{h}_{-\vec{k}}^{i n t}\right\rangle_{0} \Phi_{h h}^{-1}(\vec{k}) \hat{h}_{\vec{k}}^{i n t}+\sum_{\vec{k}}^{\prime} \int d \vec{p} \int d \vec{p}^{\prime}\left\langle\hat{A}_{\vec{k}} \hat{n}_{-\vec{k}}(\vec{p})\right\rangle_{0} \Phi_{\vec{k}}^{-1}\left(\vec{p}, \vec{p}^{\prime}\right) \hat{n}_{\vec{k}}\left(\vec{p}^{\prime}\right) .
$$

It possesses the following properties $P_{0} P_{0}=P_{0}, P_{0}\left(1-P_{0}\right)=0, P_{0} \hat{n}_{\vec{k}}(\vec{p})=\hat{n}_{\vec{k}}(\vec{p}), P_{0} \hat{h}_{\vec{k}}^{\text {int }}=$ $\hat{h}_{\vec{k}}^{i n t}$. 
In view of its own structure, the nonequilibrium distribution function (2.5) is a functional of the reduced-description parameters $\left\langle\hat{n}_{\vec{k}}(\vec{p})\right\rangle^{t},\left\langle\hat{h}_{\vec{k}}^{\text {int }}\right\rangle^{t}$, dynamic variables $\hat{n}_{\vec{k}}(\vec{p}), \hat{h}_{\vec{k}}^{\text {int }}$ along with their generalized flows (2.6). Using $\varrho\left(x^{N} ; t\right)(2.5)$ for the parameters of reduced description $f_{\vec{k}}(\vec{p} ; t)=\left\langle\hat{n}_{\vec{k}}(\vec{p})\right\rangle^{t}, h_{\vec{k}}^{\text {int }}(t)=\left\langle\hat{h}_{\vec{k}}^{\text {int }}\right\rangle^{t}$ we can obtain the following set of equations [21]:

$$
\begin{aligned}
& \frac{\partial}{\partial t} f_{\vec{k}}(\vec{p} ; t)+\frac{i \vec{k} \cdot \vec{p}}{m} f_{\vec{k}}(\vec{p} ; t)=-\frac{i \vec{k} \cdot \vec{p}}{m} n f_{0}(p) c_{2}(k) \int d \vec{p}^{\prime} f_{\vec{k}}\left(\vec{p}^{\prime} ; t\right)+i \Omega_{n h}(\vec{k} ; \vec{p}) h_{\vec{k}}^{i n t}(t) \\
& -\int d \vec{p}^{\prime} \int_{-\infty}^{t} e^{\varepsilon\left(t^{\prime}-t\right)} \varphi_{n n}\left(\vec{k} ; \vec{p}, \vec{p}^{\prime} ; t, t^{\prime}\right) f_{\vec{k}}\left(\vec{p}^{\prime} ; t^{\prime}\right) d t^{\prime}-\int_{-\infty}^{t} e^{\varepsilon\left(t^{\prime}-t\right)} \varphi_{n h}\left(\vec{k} ; \vec{p} ; t, t^{\prime}\right) h_{\vec{k}}^{i n t}\left(t^{\prime}\right) d t^{\prime}, \\
& \frac{\partial}{\partial t} h_{\vec{k}}^{i n t}(t)=\int d \vec{p}^{\prime} i \Omega_{h n}\left(\vec{k} ; \vec{p}^{\prime}\right) f_{\vec{k}}\left(\vec{p}^{\prime} ; t\right) \\
& \quad-\int d \vec{p}^{\prime} \int_{-\infty}^{t} e^{\varepsilon\left(t^{\prime}-t\right)} \varphi_{h n}\left(\vec{k} ; \vec{p}^{\prime} ; t, t^{\prime}\right) f_{\vec{k}}\left(\vec{p}^{\prime} ; t^{\prime}\right) d t^{\prime}-\int_{-\infty}^{t} e^{\varepsilon\left(t^{\prime}-t\right)} \varphi_{h h}\left(\vec{k} ; t, t^{\prime}\right) h_{\vec{k}}^{i n t}\left(t^{\prime}\right) d t^{\prime}
\end{aligned}
$$

Here, $i \Omega_{n h}(\vec{k} ; \vec{p}), i \Omega_{h n}(\vec{k} ; \vec{p})$ are the normalized static correlation functions

$$
i \Omega_{n h}(\vec{k} ; \vec{p})=\left\langle\dot{\hat{n}}_{\vec{k}}(\vec{p}) \hat{h}_{-\vec{k}}^{i n t}\right\rangle_{0} \Phi_{h h}^{-1}(\vec{k}), \quad i \Omega_{h n}(\vec{k} ; \vec{p})=\int d \vec{p}^{\prime}\left\langle\dot{\hat{h}}_{\vec{k}}^{i n t} \hat{n}_{-\vec{k}}\left(\vec{p}^{\prime}\right)\right\rangle_{0} \Phi_{\vec{k}}^{-1}\left(\vec{p}^{\prime}, \vec{p}\right)
$$

and

$$
\begin{gathered}
\varphi_{n n}\left(\vec{k} ; \vec{p}, \vec{p}^{\prime} ; t, t^{\prime}\right)=\int d \vec{p}^{\prime \prime}\left\langle I_{n}(\vec{k} ; \vec{p}) T_{0}\left(t, t^{\prime}\right) I_{n}\left(-\vec{k} ; \vec{p}^{\prime \prime}\right)\right\rangle_{0} \Phi_{\vec{k}}^{-1}\left(\vec{p}^{\prime \prime}, \vec{p}^{\prime}\right), \\
\varphi_{h n}\left(\vec{k} ; \vec{p} ; t, t^{\prime}\right)=\int d \vec{p}^{\prime}\left\langle I_{h}^{i n t}(\vec{k}) T_{0}\left(t, t^{\prime}\right) I_{n}\left(-\vec{k} ; \vec{p}^{\prime}\right)\right\rangle_{0} \Phi_{\vec{k}}^{-1}\left(\vec{p}^{\prime}, \vec{p}\right), \\
\varphi_{h n}\left(\vec{k} ; \vec{p} ; t, t^{\prime}\right)=\left\langle I_{n}(\vec{k} ; \vec{p}) T_{0}\left(t, t^{\prime}\right) I_{h}^{i n t}(-\vec{k})\right\rangle_{0} \Phi_{h h}^{-1}(\vec{k}), \\
\varphi_{h h}\left(\vec{k} ; \vec{p} ; t, t^{\prime}\right)=\left\langle I_{h}^{i n t}(\vec{k}) T_{0}\left(t, t^{\prime}\right) I_{h}^{i n t}(-\vec{k})\right\rangle_{0} \Phi_{h h}^{-1}(\vec{k})
\end{gathered}
$$

are the generalized transport kernels (memory functions) describing kinetic and hydrodynamic processes. The set of equations (2.8) and (2.9) is closed with respect to the parameters of reduced description $f_{\vec{k}}(\vec{p} ; t), h_{\vec{k}}^{\text {int }}(t)$. If in this set of equations one formally puts $\hat{h}_{\vec{k}}^{\text {int }}=0$, then we obtain the kinetic equation for $f_{\vec{k}}(\vec{p} ; t)$ :

$$
\begin{gathered}
\frac{\partial}{\partial t} f_{\vec{k}}(\vec{p} ; t)+\frac{i \vec{k} \cdot \vec{p}}{m} f_{\vec{k}}(\vec{p} ; t)=-\frac{i \vec{k} \cdot \vec{p}}{m} n f_{0}(p) c_{2}(k) \int d \vec{p}^{\prime} f_{\vec{k}}\left(\vec{p}^{\prime} ; t\right) \\
-\int d \vec{p}^{\prime} \int_{-\infty}^{t} e^{\varepsilon\left(t^{\prime}-t\right)} \varphi_{n n}^{\prime}\left(\vec{k} ; \vec{p}, \vec{p}^{\prime} ; t, t^{\prime}\right) f_{\vec{k}}\left(\vec{p}^{\prime} ; t^{\prime}\right) d t^{\prime} .
\end{gathered}
$$

This is true when the contribution form the potential energy is considerably smaller than the averaged kinetic energy (e.g. in the case of gases or weakly coupled liquids). The equation (2.12) was obtained for the first time by means of the Mori projection operators method 
in [34, 35, 37]. Therein the basic parameter of the reduced description was a nonequilibrium one-particle distribution function $f_{\vec{k}}(\vec{p} ; t)$ which corresponds to the microscopic phase density $\hat{n}_{\vec{k}}(\vec{p})$ (the Klimontovich function). In this case the memory function $\varphi_{n n}^{\prime}\left(\vec{k} ; \vec{p}, \vec{p}^{\prime} ; t, t^{\prime}\right)$ has the following structure:

$$
\varphi_{n n}^{\prime}\left(\vec{k} ; \vec{p}, \vec{p}^{\prime} ; t, t^{\prime}\right)=\int d \vec{p}^{\prime \prime}\left\langle I_{n}^{0}(\vec{k}, \vec{p}) T_{0}^{\prime}\left(t, t^{\prime}\right) I_{n}^{0}\left(-\vec{k}, \vec{p}^{\prime \prime}\right)\right\rangle_{0} \Phi_{\vec{k}}^{-1}\left(\vec{p}^{\prime \prime}, \vec{p}^{\prime}\right),
$$

where $I_{n}^{0}(\vec{k}, \vec{p})=\left(1-P_{0}^{\prime}\right) \dot{\hat{n}}_{\vec{k}}(\vec{p})$ is the generalized flow, $P_{0}^{\prime}$ is the Mori operator, introduced in $[34,35,37]$

$$
P_{0}^{\prime} \hat{A}_{\vec{k}^{\prime}}=\sum_{\vec{k}}^{\prime} \int d \vec{p} \int d \vec{p}^{\prime}\left\langle\hat{A}_{\vec{k}^{\prime}} \hat{n}_{-\vec{k}^{\prime}}\left(\vec{p}^{\prime}\right)\right\rangle_{0} \Phi_{\vec{k}}^{-1}\left(\vec{p}^{\prime}, \vec{p}\right) \hat{n}_{\vec{k}}(\vec{p}),
$$

and $T_{0}^{\prime}\left(t, t^{\prime}\right)$ is the corresponding evolution operator with regard to projection. Based on the kinetic equation (2.12) the investigations of the dynamic structure factor, transverse and longitudinal current time correlation functions, diffusion and viscosity coefficients for dense gases and liquids where carried out [2, 10, 34 46]. In particular, in Mazenko's papers the linearized Boltzmann-Enskog equation was obtained by means of expansion of the memory functions $\varphi_{n n}^{\prime}\left(\vec{k} ; \vec{p}, \vec{p}^{\prime} ; t, t^{\prime}\right)$ in density. For the case of weak coupling, the equation of FokkerPlanck type was obtained. However, the main drawback of kinetic equation (2.12) consists in its inconsistency with the total energy conservation law, especially for dense gases and liquids, when the contribution of the potential energy into the thermodynamic functions and transport coefficients is determinant. In [2] this drawback was studied in detail. There the investigations of the dynamic structure factor at intermediate values of wave-vector $\vec{k}$ and frequency $\omega$ for simple liquids were carried out by using the Mori projection operators method for the reduced-description parameters $\hat{n}_{\vec{k}}(\vec{p}), \hat{\varepsilon}_{\vec{k}}$. Contrary to the transport equations presented in [2], our set of equations (2.8) and (2.9) is constructed on the orthogonal dynamic variables $\hat{n}_{\vec{k}}(\vec{p}), \hat{h}_{\vec{k}}^{\text {int }}$. Therefore, "kinetic" and "hydrodynamic" contributions are separated and correlation between them is described by the generalized memory functions (2.11). It is important to reveal their inner structure.

\section{MEMORY FUNCTIONS OF A CONSISTENT DESCRIPTION OF KINETIC AND HYDRODYNAMIC PROCESSES}

In order to study the structure of the memory functions (2.11) let us look at the form of the corresponding generalized flows (2.6) on which the memory functions are built. In 
particular, let us take into consideration the fact that

$$
\dot{\hat{n}}_{\vec{k}}(\vec{p})=i L_{N} \hat{n}_{\vec{k}}(\vec{p})=-\frac{i \vec{k}}{m} \cdot \hat{\vec{\jmath}}(\vec{p})+\frac{\partial}{\partial \vec{p}} \cdot \vec{F}_{\vec{k}}(\vec{p})
$$

where

$$
\hat{\vec{\jmath}} \overrightarrow{\vec{p}}(\vec{p})=\sum_{j=1}^{N} \vec{p}_{j} e^{-i \vec{k} \cdot \vec{r}_{j}} \delta\left(\vec{p}-\vec{p}_{j}\right)
$$

is the momentum density in the $(\vec{k}, \vec{p})$ space and

$$
\vec{F}_{\vec{k}}(\vec{p})=\frac{1}{2} \sum_{j \neq l} \frac{\partial}{\partial \vec{r}_{j}} \Phi\left(\left|\vec{r}_{j}-\vec{r}_{l}\right|\right) \delta\left(\vec{p}-\vec{p}_{j}\right) e^{-i \vec{k} \cdot \vec{r}_{j}}
$$

The action of the Mori projection operator $P_{0}$ on $\dot{\hat{n}}_{\vec{k}}(\vec{p})$ can be presented as

$$
P_{0} \dot{\hat{n}}_{\vec{k}}(\vec{p})=-\frac{\beta}{m} \vec{p} \cdot \vec{\Phi}_{F h}(\vec{k}) \hat{h}_{\vec{k}}^{i n t} f_{0}(p)-\frac{i \vec{k}}{m} \cdot \vec{p} \hat{n}_{\vec{k}}(\vec{p})
$$

where

$$
\vec{\Phi}_{F h}(\vec{k})=\left\langle\vec{F}_{\vec{k}} \hat{h}_{\vec{k}}^{i n t}\right\rangle_{0} \Phi_{h h}^{-1}(\vec{k})
$$

Taking into account (2.4) we write down the memory function $\varphi_{n n}\left(\vec{k} ; \vec{p}, \vec{p}^{\prime} ; t, t^{\prime}\right)$ in the following form

$$
\begin{aligned}
& \varphi_{n n}\left(\vec{k} ; \vec{p}, \vec{p}^{\prime} ; t, t^{\prime}\right)=\int d \vec{p}^{\prime \prime}\left\langle I_{n}(\vec{k} ; \vec{p}) T_{0}\left(t, t^{\prime}\right) I_{n}(-k ; \overrightarrow{\vec{p}})\right\rangle_{0}\left\{\frac{\delta\left(\vec{p}^{\prime}-\vec{p}^{\prime}\right)}{n f_{0}\left(p^{\prime}\right)}-c_{2}(k)\right\} \\
& \quad=\left\langle I_{n}(\vec{k} ; \vec{p}) T_{0}\left(t, t^{\prime}\right) I_{n}(-k ; \vec{p})\right\rangle_{0} \frac{1}{n f_{0}\left(p^{\prime}\right)}-\int d \vec{p}^{\prime \prime}\left\langle I_{n}(\vec{k} ; \vec{p}) T_{0}\left(t, t^{\prime}\right) I_{n}(-k ; \vec{p} \vec{p})\right\rangle_{0} c_{2}(k) .
\end{aligned}
$$

The second term in the right-hand side of (3.6) is equal to zero, because

$$
\int d \vec{p} I_{n}(\vec{k} ; \vec{p})=0
$$

The transport kernel $\varphi_{n n}\left(\vec{k} ; \vec{p}, \vec{p}^{\prime} ; t, t^{\prime}\right)$ enters into the kinetic equation (2.8) as the term $\int \varphi_{n n}\left(\vec{k} ; \vec{p}, \vec{p}^{\prime} ; t, t^{\prime}\right) f_{\vec{k}}\left(\vec{p}^{\prime} ; t\right) d \vec{p}^{\prime}$. Taking into account (3.6) and (3.7) we can write the last one in the form

$$
\begin{aligned}
& \int d \vec{p}^{\prime} \varphi_{n n}\left(\vec{k} ; \vec{p}, \vec{p}^{\prime} ; t, t^{\prime}\right) f_{\vec{k}}\left(\vec{p}^{\prime} ; t\right)=\int d \vec{p}^{\prime}\left\{\bar{\varphi}_{\jmath \jmath}\left(\vec{k} ; \vec{p}, \vec{p}^{\prime} ; t, t^{\prime}\right)\right. \\
& \left.-\frac{\partial}{\partial \vec{p}} \cdot \varphi_{F F}\left(\vec{k} ; \vec{p}, \vec{p}^{\prime} ; t, t^{\prime}\right) \cdot\left(\frac{\beta}{m n f_{0}\left(p^{\prime}\right)} \vec{p}^{\prime}-\frac{\partial}{\partial \vec{p}}\right)\right\} f_{\vec{k}}\left(\vec{p}^{\prime} ; t^{\prime}\right)-\bar{\varphi}_{n \jmath}^{(2)}\left(\vec{k} ; \vec{p} ; t, t^{\prime}\right) \cdot\left\langle\hat{\vec{J}}_{\vec{k}}\right\rangle^{t},
\end{aligned}
$$


where the second term has the structure of the generalized Fokker-Planck operator containing the generalized friction coefficient $\varphi_{F F}\left(\vec{k} ; \vec{p}, \vec{p}^{\prime} ; t, t^{\prime}\right)$ in the spatially-impulse space.

$$
\begin{gathered}
\bar{\varphi}_{\jmath \jmath}\left(\vec{k} ; \vec{p}, \vec{p}^{\prime} ; t, t^{\prime}\right)=\frac{\vec{k}}{m} \cdot \varphi_{\jmath \jmath}\left(\vec{k} ; \vec{p}, \vec{p}^{\prime} ; t, t^{\prime}\right) \cdot \frac{\vec{k}}{m} \\
-\frac{i \vec{k}}{m} \cdot \varphi_{\jmath F}\left(\vec{k} ; \vec{p}, \vec{p}^{\prime} ; t, t^{\prime}\right) \cdot \frac{\partial}{\partial \vec{p}^{\prime}}+\frac{\partial}{\partial \vec{p}} \cdot \varphi_{F \jmath}\left(\vec{k} ; \vec{p}, \vec{p}^{\prime} ; t, t^{\prime}\right) \cdot \frac{i \vec{k}}{m}+\bar{\varphi}_{n n}^{(1)}\left(\vec{k} ; \vec{p}, \vec{p}^{\prime} ; t, t^{\prime}\right), \\
\varphi_{F F}\left(\vec{k} ; \vec{p}, \vec{p}^{\prime} ; t, t^{\prime}\right)=\left\langle\vec{F}_{\vec{k}}(\vec{p}) T_{0}\left(t, t^{\prime}\right) \vec{F}_{-\vec{k}}\left(\vec{p}^{\prime}\right)\right\rangle_{0}, \quad \varphi_{\jmath \jmath}\left(\vec{k} ; \vec{p}, \vec{p}^{\prime} ; t, t^{\prime}\right)=\left\langle\hat{\vec{\jmath}}_{\vec{k}}(\vec{p}) T_{0}\left(t, t^{\prime}\right) \hat{\vec{\jmath}}_{-\vec{k}}\left(\vec{p}^{\prime}\right)\right\rangle_{0}, \\
\varphi_{\jmath F}\left(\vec{k} ; \vec{p}, \vec{p}^{\prime} ; t, t^{\prime}\right)=\left\langle\hat{\vec{\jmath}_{\vec{k}}}(\vec{p}) T_{0}\left(t, t^{\prime}\right) \vec{F}_{-\vec{k}}\left(\vec{p}^{\prime}\right)\right\rangle_{0}, \quad \varphi_{F \jmath}\left(\vec{k} ; \vec{p}, \vec{p}^{\prime} ; t, t^{\prime}\right)=\left\langle\vec{F}_{\vec{k}}(\vec{p}) T_{0}\left(t, t^{\prime}\right) \hat{\vec{\jmath}}-\vec{k}\left(\vec{p}^{\prime}\right)\right\rangle_{0},
\end{gathered}
$$

moreover,

$$
\int d \vec{p} \int d \vec{p}^{\prime} \varphi_{\jmath \jmath}\left(\vec{k} ; \vec{p}, \vec{p}^{\prime} ; t, t^{\prime}\right)=D\left(\vec{k} ; t, t^{\prime}\right)
$$

is the generalized coefficient of diffusion of particles and $\varphi_{\jmath \jmath}\left(\vec{k} ; \vec{p}, \vec{p}^{\prime} ; t, t^{\prime}\right)$ the generalized diffusion coefficient in momentum space.

$$
\int d \vec{p} \int d \vec{p}^{\prime} \varphi_{F F}\left(\vec{k} ; \vec{p}, \vec{p}^{\prime} ; t, t^{\prime}\right)=\xi\left(\vec{k} ; t, t^{\prime}\right)
$$

is the generalized friction coefficient and $\varphi_{F F}\left(\vec{k} ; \vec{p}, \vec{p}^{\prime} ; t, t^{\prime}\right)$ the generalized friction coefficient in momentum space.

The structure of functions $\bar{\varphi}_{n n}^{(1)}\left(\vec{k} ; \vec{p}, \vec{p}^{\prime} ; t, t^{\prime}\right)$ and $\varphi_{n j}^{(2)}\left(\vec{k} ; \vec{p} ; t, t^{\prime}\right)$ is presented in the Appendix. In particular, they are determined by the time correlation functions built on the set of dynamic variables $\hat{n}_{\vec{k}}(\vec{p}), \hat{h}_{\vec{k}}^{i n t}, \hat{\vec{J}}_{\vec{k}}(\vec{p})$ and $\vec{F}_{\vec{k}}(\vec{p})$.

In the kinetic equation (2.8) the transport kernel $\varphi_{n h}\left(\vec{k} ; \vec{p} ; t, t^{\prime}\right)$ describes dynamic correlations between the kinetic and hydrodynamic processes. Performing the action of operators $\left(1-P_{0}\right)$ and $i L_{N}$ as well as taking into account (3.4) and

$$
P_{0} \dot{\hat{h}}_{\vec{k}}^{i n t}={\overrightarrow{\Phi^{\prime}}}_{h F}(\vec{k}) \cdot \hat{\vec{\jmath}}_{\vec{k}}, \quad \vec{\Phi}_{h F}^{\prime}(\vec{k})=\left\langle\hat{h}_{\vec{k}}^{i n t} \vec{F}_{-\vec{k}}\right\rangle_{0} \frac{\beta}{m n},
$$

the kernel $\varphi_{n h}\left(\vec{k} ; \vec{p} ; t, t^{\prime}\right)$ can be presented as

$$
\begin{aligned}
& \varphi_{n h}\left(\vec{k} ; \vec{p} ; t, t^{\prime}\right)=-\frac{i \vec{k}}{m} \cdot \bar{\varphi}_{\jmath \dot{h}}\left(\vec{k} ; \vec{p} ; t, t^{\prime}\right)+\frac{\partial}{\partial \vec{p}} \cdot \bar{\varphi}_{F \dot{h}}\left(\vec{k} ; \vec{p} ; t, t^{\prime}\right)+\frac{\beta}{m} \vec{p} \cdot \vec{\Phi}_{F h}(\vec{k}) f_{0}(p) \bar{\varphi}_{h \dot{h}}\left(\vec{k} ; t, t^{\prime}\right) \\
& \quad+\frac{i \vec{k}}{m} \cdot \vec{p} \bar{\varphi}_{n \dot{h}}\left(\vec{k} ; \vec{p} ; t, t^{\prime}\right)+\frac{i \vec{k}}{m} \cdot \varphi_{\jmath \jmath}\left(\vec{k} ; \vec{p} ; t, t^{\prime}\right) \cdot \vec{\Phi}_{h F}^{\prime}(\vec{k})-\frac{\partial}{\partial \vec{p}} \cdot \varphi_{F \jmath}\left(\vec{k} ; \vec{p} ; t, t^{\prime}\right) \vec{\Phi}_{h F}^{\prime}(\vec{F}) \\
& \quad-\frac{\beta}{m} \vec{p} \cdot \vec{\Phi}_{F h}(\vec{k}) f_{0}(p) \varphi_{h \jmath}\left(\vec{k} ; t, t^{\prime}\right) \cdot \vec{\Phi}_{h F}^{\prime}(\vec{k})-\frac{i \vec{k}}{m} \cdot \vec{p} \varphi_{n \jmath}\left(\vec{k} ; \vec{p} ; t, t^{\prime}\right) \vec{\Phi}_{h F}^{\prime}(\vec{k}),
\end{aligned}
$$


The structure of the correlation functions entering this equation is presented in the Appendix as well. The correlation functions $\varphi_{\jmath \jmath}\left(\vec{k} ; \vec{p} ; t, t^{\prime}\right), \varphi_{F \jmath}\left(\vec{k} ; \vec{p} ; t, t^{\prime}\right), \varphi_{h \jmath}\left(\vec{k} ; \vec{p} ; t, t^{\prime}\right)$, and $\varphi_{n \jmath}\left(\vec{k} ; \vec{p} ; t, t^{\prime}\right)$ have the structure similar to (A.2).

From the structure of the transport kernels (3.8), (3.9), (A.1), (A.3) and (3.13) in the kinetic equation (2.8) for a nonequilibrium one-particle distribution function one can see that the contributions of the hydrodynamic processes are described, besides $\hat{h}_{\vec{k}}^{\text {int }}$, by the moments $\int d \vec{p} f_{\vec{k}}(\vec{p} ; t)=n_{\vec{k}}(t)=\left\langle\hat{n}_{\vec{k}}\right\rangle^{t}$ and $\int d \vec{p} \vec{p} f_{\vec{k}}(\vec{p} ; t)=\left\langle\hat{\vec{j}}_{\vec{k}}\right\rangle^{t}$.

As in the case of equation (2.8), let us find the inner structure of transport kernels in equation (2.9) for the average value of the potential part of the enthalpy. In particular, taking into account (3.13), for $\varphi_{h h}\left(\vec{k} ; t, t^{\prime}\right)$ we obtain:

$$
\begin{gathered}
\varphi_{h h}\left(\vec{k} ; t, t^{\prime}\right)=\bar{\varphi}_{\dot{h} \dot{h}}^{(0)}\left(\vec{k} ; t, t^{\prime}\right)-\vec{\Phi}_{h F}^{\prime}(\vec{k}) \cdot \bar{\varphi}_{j \dot{h}}^{(0)}\left(\vec{k} ; t, t^{\prime}\right) \\
-\bar{\varphi}_{\dot{h}_{\jmath}}\left(\vec{k} ; t, t^{\prime}\right) \cdot \vec{\Phi}_{h F}^{\prime}(\vec{k})+\vec{\Phi}_{h F}^{\prime}(\vec{k}) \cdot \varphi_{\jmath \jmath}^{(0)}\left(\vec{k} ; t, t^{\prime}\right) \cdot \vec{\Phi}_{F h}(\vec{k}) . \\
\bar{\varphi}_{\dot{h} \dot{h}}^{(0)}\left(\vec{k} ; t, t^{\prime}\right)=\left\langle\dot{\hat{h}}_{\vec{k}}^{i n t} T_{0}\left(t, t^{\prime}\right) \dot{\hat{h}}_{-\vec{k}}^{i n t}\right\rangle_{0} \Phi_{h h}^{-1}(\vec{k}), \bar{\varphi}_{\jmath \dot{h}}^{(0)}\left(\vec{k} ; t, t^{\prime}\right)=\left\langle\hat{\vec{\jmath}}_{\vec{k}} T_{0}\left(t, t^{\prime}\right) \dot{\hat{h}}_{\vec{k}}^{i n t}\right\rangle_{0} \Phi_{h h}^{-1}(\vec{k}), \quad \\
\bar{\varphi}_{\dot{h}_{\jmath}}\left(\vec{k} ; t, t^{\prime}\right)=\Phi_{h h}^{-1}(\vec{k})\left\langle\dot{\hat{h}}_{\vec{k}}^{i n t} T_{0}\left(t, t^{\prime}\right) \hat{\vec{\jmath}}_{\vec{k}}\right\rangle_{0}, \quad \varphi_{\jmath \jmath}^{(0)}\left(\vec{k} ; t, t^{\prime}\right)=\left\langle\hat{\vec{\jmath}}_{\vec{k}} T_{0}\left(t, t^{\prime}\right) \hat{\vec{\jmath}}_{\vec{k}}\right\rangle_{0}=D\left(\vec{k} ; t, t^{\prime}\right)
\end{gathered}
$$

that is, $\varphi_{\jmath \jmath}^{(0)}\left(\vec{k} ; t, t^{\prime}\right)$ is the generalized diffusion coefficient (3.11), whereas, $\bar{\varphi}_{\dot{h} \dot{h}}^{(0)}\left(\vec{k} ; t, t^{\prime}\right)$ determines a potential part of the generalized heat-conductivity coefficient. Taking into account (3.4), (3.14) the transport kernel $\varphi_{h n}\left(\vec{k} ; \vec{p} ; t, t^{\prime}\right)$ entering (2.91) as $\int d \vec{p}^{\prime} \varphi_{h n}\left(\vec{k} ; \vec{p}^{\prime} ; t, t^{\prime}\right) f_{\vec{k}}\left(\vec{p}^{\prime} ; t^{\prime}\right)$ can be presented in the following way

$$
\begin{aligned}
& \int d \vec{p}^{\prime} \varphi_{h n}\left(\vec{k} ; \vec{p}^{\prime} ; t, t^{\prime}\right) f_{\vec{k}}\left(\vec{p}^{\prime} ; t^{\prime}\right)=\frac{i \vec{k}}{m} \cdot \int d \vec{p}^{\prime} W_{h n}\left(\vec{k} ; \vec{p}^{\prime} ; t, t^{\prime}\right) \frac{1}{n f_{0}\left(p^{\prime}\right)} f_{\vec{k}}\left(\vec{p}^{\prime} ; t\right) \\
& -\int d \vec{p}^{\prime} W_{h F}\left(\vec{k} ; \vec{p}^{\prime} ; t, t^{\prime}\right) \frac{1}{n f_{0}\left(p^{\prime}\right)} \cdot\left(\beta \frac{\vec{p}^{\prime}}{m n}-\frac{\partial}{\partial \vec{p}^{\prime}}\right) f_{\vec{k}}\left(\vec{p}^{\prime} ; t^{\prime}\right)+W_{h j}\left(\vec{k} ; t, t^{\prime}\right) \frac{\beta}{m n} \cdot\langle\hat{\vec{j}} \vec{k}\rangle^{t^{\prime}},
\end{aligned}
$$

where

$$
\begin{gathered}
W_{h n}\left(\vec{k} ; \vec{p}^{\prime} ; t, t^{\prime}\right)=\varphi_{h_{\jmath}}\left(\vec{k} ; \vec{p}^{\prime} ; t, t^{\prime}\right) \\
-\varphi_{h n}\left(\vec{k} ; \vec{p}^{\prime} ; t, t^{\prime}\right) \cdot \vec{p}^{\prime}-\vec{\Phi}_{h F}^{\prime}(\vec{k}) \varphi_{\jmath \jmath}\left(\vec{k} ; \vec{p}^{\prime} ; t, t^{\prime}\right)+\vec{\Phi}_{h F}^{\prime}(\vec{k}) \varphi_{\jmath n}\left(\vec{k} ; \vec{p}^{\prime} ; t, t^{\prime}\right) \cdot \vec{p}^{\prime}, \\
W_{h F}\left(\vec{k} ; \vec{p}^{\prime} ; t, t^{\prime}\right)=\varphi_{h F}\left(\vec{k} ; \vec{p}^{\prime} ; t, t^{\prime}\right)+\vec{\Phi}_{h F}^{\prime}(\vec{k}) \varphi_{\jmath F}\left(\vec{k} ; \vec{p}^{\prime} ; t, t^{\prime}\right), \\
W_{h \jmath}\left(\vec{k} ; t, t^{\prime}\right)=\varphi_{h_{h}}\left(\vec{k} ; t, t^{\prime}\right) \vec{\Phi}_{h F}^{\prime}(\vec{k})-\vec{\Phi}_{h F}^{\prime}(\vec{k}) \varphi_{\jmath h}\left(\vec{k} ; t, t^{\prime}\right) \vec{\Phi}_{h F}^{\prime}(\vec{k})
\end{gathered}
$$


are the transport kernels, formed by the time correlation functions of type of (A.5) and (3.16). Taking into account the structure of the memory functions (3.8), (3.14), (3.15) and (3.18), we present the set of equations (2.8), (2.9) in the form

$$
\begin{aligned}
& \frac{\partial}{\partial t} f_{\vec{k}}(\vec{p} ; t)+\frac{i \vec{k}}{m} \cdot \vec{p} f_{\vec{k}}(\vec{p} ; t)= \\
& -\frac{i \vec{k}}{m} \cdot \overrightarrow{p n} f_{0}(p) c_{2}(\vec{k}) \int d \vec{p}^{\prime} f_{\vec{k}}\left(\vec{p}^{\prime} ; t\right)+i \Omega_{n h}(\vec{k} ; \vec{p}) h_{\vec{k}}^{i n t}(t) \\
& -\int d \vec{p}^{\prime} \int_{-\infty}^{t} e^{\varepsilon\left(t^{\prime}-t\right)}\left\{\varphi_{\jmath \jmath}\left(\vec{k} ; \vec{p}, \vec{p}^{\prime} ; t, t^{\prime}\right)\right. \\
& \left.-\frac{\partial}{\partial \vec{p}} \cdot \varphi_{F F}\left(\vec{k} ; \vec{p}, \vec{p}^{\prime} ; t, t^{\prime}\right) \cdot\left(\frac{\beta \vec{p}^{\prime}}{m n f_{0}\left(p^{\prime}\right)}-\frac{\partial}{\partial \vec{p}^{\prime}}\right)\right\} f_{\vec{k}}\left(\vec{p}^{\prime} ; t^{\prime}\right) d t^{\prime} \\
& +\int_{-\infty}^{t} e^{\varepsilon\left(t^{\prime}-t\right)} \varphi_{n \jmath}^{(2)}\left(\vec{k} ; \vec{p}, ; t, t^{\prime}\right) \cdot \vec{\jmath}_{\vec{k}}(t) \\
& +\int_{-\infty}^{t} e^{\varepsilon\left(t^{\prime}-t\right)}\left\{\frac{i \vec{k}}{m} \cdot W_{n h}\left(\vec{k} ; \vec{p} ; t, t^{\prime}\right)-\frac{\partial}{\partial \vec{p}} \cdot W_{F h}\left(\vec{k} ; \vec{p} ; t, t^{\prime}\right)\right. \\
& \left.-\frac{\beta}{m} f_{0}(p) \vec{p} \cdot W_{F \dot{h}}\left(\vec{k} ; \vec{p} ; t, t^{\prime}\right)\right\} h_{\vec{k}}^{i n t}\left(t^{\prime}\right) d t^{\prime}, \\
& \frac{\partial}{\partial t} h_{\vec{k}}^{i n t}(t)=\int d \vec{p}^{\prime} i \Omega_{h n}\left(k ; \vec{p} \vec{p}^{\prime} f_{\vec{k}}\left(\vec{p}^{\prime} ; t\right)\right. \\
& -\int_{-\infty}^{t} e^{\varepsilon\left(t^{\prime}-t\right)}\left\{\bar{\varphi}_{\dot{h} \dot{h}}^{(0)}\left(\vec{k} ; t, t^{\prime}\right)-\vec{\Phi}_{h F}^{\prime}(\vec{k}) \cdot \bar{\varphi}_{\jmath h}^{(0)}\left(\vec{k} ; t, t^{\prime}\right)\right. \\
& \left.-\bar{\varphi}_{h \jmath}\left(\vec{k} ; t, t^{\prime}\right) \cdot \vec{\Phi}_{h F}^{\prime}(\vec{k})+\vec{\Phi}_{h F}^{\prime}(\vec{k}) \cdot \bar{\varphi}_{\jmath \jmath}^{(0)}\left(\vec{k} ; t, t^{\prime}\right) \cdot \vec{\Phi}_{F h}(\vec{k})\right\} h_{\vec{k}}^{i n t}\left(t^{\prime}\right) d t^{\prime} \\
& -\frac{i \vec{k}}{m} \cdot \int d \vec{p}^{\prime} \int_{-\infty}^{t} e^{\varepsilon\left(t^{\prime}-t\right)} W_{h n}\left(\vec{k} ; \vec{p}^{\prime} ; t, t^{\prime}\right) \frac{1}{n f_{0}\left(p^{\prime}\right)} f_{\vec{k}}\left(\vec{p}^{\prime} ; t^{\prime}\right) d t^{\prime} \\
& +\int d \vec{p}^{\prime} \int_{-\infty}^{t} e^{\varepsilon\left(t^{\prime}-t\right)} W_{h F}\left(\vec{k} ; \vec{p}^{\prime} ; t, t^{\prime}\right) \frac{1}{n f_{0}\left(p^{\prime}\right)} \cdot\left(\frac{\beta}{m n} \vec{p}^{\prime}-\frac{\partial}{\partial \vec{p}^{\prime}}\right) f_{\vec{k}}\left(\vec{p}^{\prime} ; t^{\prime}\right) d t^{\prime} \\
& -\int_{-\infty}^{t} e^{\varepsilon\left(t^{\prime}-t\right)} W_{h_{\jmath}}\left(\vec{k} ; t, t^{\prime}\right) \frac{\beta}{m n} \cdot \vec{\jmath}_{\vec{k}}\left(t^{\prime}\right) d t^{\prime},
\end{aligned}
$$

where in the first equation the transport kernels have the following structure:

$$
\begin{gathered}
W_{n \dot{h}}\left(\vec{k} ; \vec{p} ; t, t^{\prime}\right)=\bar{\varphi}_{\jmath \dot{h}}\left(\vec{k} ; \vec{p} ; t, t^{\prime}\right) \\
-\vec{p} \cdot \bar{\varphi}_{n \dot{h}}\left(\vec{k} ; \vec{p} ; t, t^{\prime}\right)-\varphi_{\jmath \jmath}\left(\vec{k} ; \vec{p} ; t, t^{\prime}\right) \cdot \vec{\Phi}_{h F}^{\prime}(\vec{k})+\vec{p} \cdot \varphi_{n \jmath}\left(\vec{k} ; \vec{p} ; t, t^{\prime}\right) \cdot \vec{\Phi}_{h F}^{\prime}(\vec{k}), \\
W_{F h}\left(\vec{k} ; \vec{p} ; t, t^{\prime}\right)=\bar{\varphi}_{F h}\left(\vec{k} ; \vec{p} ; t, t^{\prime}\right)-\varphi_{F \jmath}\left(\vec{k} ; \vec{p} ; t, t^{\prime}\right) \cdot \vec{\Phi}_{h F}^{\prime}(\vec{k}), \\
W_{F \dot{h}}\left(\vec{k} ; \vec{p} ; t, t^{\prime}\right)=\vec{\Phi}_{F h}(\vec{k}) \cdot \varphi_{h \dot{h}}\left(\vec{k} ; t, t^{\prime}\right)-\vec{\Phi}_{F h}(\vec{k}) \cdot \varphi_{h \jmath}\left(\vec{k} ; t, t^{\prime}\right) \cdot \vec{\Phi}_{h F}^{\prime}(\vec{k}) .
\end{gathered}
$$


The transport equations (3.19), (3.20) are functionally connected concerning the basic parameters of reduced description $f_{\vec{k}}(\vec{p} ; t), h_{\vec{k}}^{\text {int }}(t)$. However, the equations contain the average values of densities of particles number $\left\langle\hat{n}_{\vec{k}}\right\rangle^{t}$ and momentum $\left\langle\hat{\vec{j}}_{\vec{k}}\right\rangle^{t}$, which, generally speaking, are the hydrodynamic variables. Integrating the equation (3.19) over momentum, we obtain the equation for $n_{\vec{k}}(t)$

$$
\frac{\partial}{\partial t} n_{\vec{k}}(t)+\frac{i \vec{k}}{m} \cdot \vec{\jmath}_{\vec{k}}(t)=0
$$

that represents the conservation law for average value of number of particles.

It is worth noting that the generalized diffusion and friction coefficients (3.9) $-(3.12)$ in the phase space together with the generalized coefficients (3.15)-(3.16), the diffusion coefficient $\varphi_{\jmath \jmath}^{(0)}\left(\vec{k} ; t, t^{\prime}\right)$, the potential part of the generalized heat conductivity coefficient $\bar{\varphi}_{\dot{h} \dot{h}}^{(0)}\left(\vec{k} ; t, t^{\prime}\right)$ as well as together with coefficients $\bar{\varphi}_{j \dot{h}}^{(0)}\left(\vec{k} ; t, t^{\prime}\right)$ and $\bar{\varphi}_{\dot{h}_{\jmath}}\left(\vec{k} ; t, t^{\prime}\right)$ describing cross-correlations between viscous and heat processes all enter the set of transport equations (3.19), (3.20).

In contrast by the equations of molecular hydrodynamics [10, 47], in this approach, the viscosity processes in the system are described by means of the generalized coefficients of diffusion and friction of particles in the phase space. Such a level of description is very important, in particular, in the case of molecular liquids, molecules of which possess their own structure and are sensitive to the process of momentum transfer when interacting. Another significant feature of these equations is connected with the averaged value of the potential part of the enthalpy density $h_{\vec{k}}^{i n t}(t)$. In the case when Fourier transform of the long-range part of the potential of interaction $\Phi\left(\left|\vec{r}_{l j}\right|\right)$ exists, the potential part of the energy density $\hat{\varepsilon}_{\vec{k}}^{i n t}$ can be expressed via the Fourier-components of particles number density:

$$
\hat{\varepsilon}_{\vec{k}}^{i n t}=\frac{1}{2} \sum_{\vec{q}} \nu(q) \hat{n}_{\vec{q}+\vec{k}} \hat{n}_{-\vec{q}}
$$

where $\nu(q)$ is the Fourier transform of the pair potential of interaction. Taking into account (3.22) the averaged value of the potential part of the enthalpy density can be presented as follows:

$$
h_{\vec{k}}^{i n t}(t)=\frac{1}{2} \sum_{\vec{q}} \nu(q) F_{2}(\vec{q}+\vec{k},-\vec{q} ; t)+\frac{1}{2} \sum_{\vec{q}} \nu(q) S_{3}(\vec{q}+\vec{k},-\vec{q},-\vec{k}) S_{2}^{-1}(\vec{q}) n_{\vec{k}}(t) .
$$

Here, $F_{2}(\vec{q}+\vec{k},-\vec{q} ; t)=\left\langle\hat{n}_{\vec{q}+\vec{k}} \hat{n}_{-\vec{q}}\right\rangle^{t}$ is the nonequilibrium scattering function, whose Fourier transform is the nonequilibrium dynamic structure factor $S_{2}(\vec{q}+\vec{k},-\vec{q} ; \omega)$ of particles of 
the system. $S_{3}(\vec{q}+\vec{k},-\vec{q},-\vec{k})=\left\langle\hat{n}_{\vec{q}+\vec{k}} \hat{n}_{-\vec{q}} \hat{n}_{-\vec{k}}\right\rangle_{0}$ is the three-particles equilibrium structure factor. The function $S_{2}(\vec{q}+\vec{k},-\vec{q} ; t)$ is important from the point of view of describing the dynamics of neutron scattering in the system [1, 6, 30].

On the basis of the generalized transport equations (3.19), (3.20) one can obtain the corresponding set of equations for time correlation functions [33].

Let us now project the set of equations (2.8), (2.9) or (3.19), (3.20) onto the first moments of the nonequilibrium one-particle distribution function

$$
\Psi_{1}(\vec{p})=1, \quad \Psi_{\alpha}(\vec{p})=\sqrt{2} p_{\alpha} / 2 k_{B} T, \quad \Psi_{\varepsilon}(\vec{p})=\sqrt{2 / 3}\left(p^{2} / 2 m k_{B} T-3 / 2\right), \quad(\alpha=x, y, z) .
$$

Then, we obtain the set of equations for the averaged values of densities of particles number $n_{\vec{k}}(t)$, momentum $\vec{\jmath}_{\vec{k}}(t)$, kinetic $h_{\vec{k}}^{\text {kin }}(t)$ and potential $h_{\vec{k}}^{i n t}(t)$ parts of enthalpy [21], where the Fourier-components of the kinetic part of enthalpy density defined as $\hat{h}_{\vec{k}}^{\text {kin }}=\hat{\varepsilon}_{\vec{k}}^{\text {kin }}-$ $\left\langle\hat{\varepsilon}_{\vec{k}}^{k i n} \hat{n}_{-\vec{k}}\right\rangle_{0}\left\langle\hat{n}_{\vec{k}} \hat{n}_{-\vec{k}}\right\rangle_{0}^{-1} \hat{n}_{\vec{k}}$. Using the Laplace transform let us represent the obtained system of equations for averages $\tilde{a}_{\vec{k}}(z)=\left\{n_{\vec{k}}(z), \vec{\jmath}_{\vec{k}}(z), h_{\vec{k}}^{\text {kin }}(z), h_{\vec{k}}^{\text {int }}(z)\right\}$ in a matrix form:

$$
z \tilde{a}_{\vec{k}}(z)-\tilde{\Sigma}^{G}(\vec{k} ; z) \tilde{a}_{\vec{k}}(z)=-\left\langle\tilde{a}_{\vec{k}}(t=0)\right\rangle^{t} .
$$

with the matrix $\tilde{\Sigma}^{G}(\vec{k} ; z)$.

$$
\tilde{\Sigma}^{G}(\vec{k} ; z)=i \tilde{\Omega}^{G}(\vec{k})+\tilde{\Pi}^{G}(\vec{k} ; z)
$$

where

$$
i \tilde{\Omega}^{G}(\vec{k})=\left(\begin{array}{llll}
0 & i \Omega_{n \jmath} & 0 & 0 \\
i \Omega_{\jmath n} & 0 & i \Omega_{\jmath h}^{k i n} & i \Omega_{\jmath h}^{i n t} \\
0 & i \Omega_{h \jmath}^{k i n} & 0 & 0 \\
0 & i \Omega_{h \jmath}^{i n t} & 0 & 0
\end{array}\right)_{(\vec{k})}
$$

is the frequency matrix, and

$$
\tilde{\Pi}^{G}(\vec{k} ; z)=\left(\begin{array}{llll}
0 & 0 & 0 & 0 \\
0 & \Pi_{\jmath \jmath} & \Pi_{\jmath h}^{k i n} & \Pi_{\jmath h}^{i n t} \\
0 & \Pi_{h \jmath}^{k i n} & \Pi_{h h}^{k i n, k i n} & \Pi_{h h}^{k i n, i n t} \\
0 & \Pi_{h \jmath}^{i n t} & \Pi_{h h}^{i n t, k i n} & \Pi_{h h}^{i n t, i n t}
\end{array}\right)_{(\vec{k} ; z)}
$$


is the matrix of transport kernels. Its elements have the following structure:

$$
\Pi_{\mu \nu}(\vec{k} ; z)=\left\langle\Psi_{\mu}\left|\tilde{\varphi}(\vec{k} ; z)+\tilde{\Sigma}(\vec{k} ; z) \mathcal{Q}[z \tilde{I}-\mathcal{Q} \tilde{\Sigma}(\vec{k} ; z) \mathcal{Q}]^{-1} \mathcal{Q} \tilde{\Sigma}(\vec{k} ; z)\right| \Psi_{\nu}\right\rangle
$$

where $\mathcal{Q}=1-\mathcal{P}$ with $\mathcal{P}$ being the projection operator constructed on the first moments $\left|\Psi_{\alpha}(\vec{p})\right\rangle$ of the nonequilibrium one-particle distribution function. This operator acts ac-

cording to the rule $\mathcal{P}\langle\Psi|=\sum_{\nu=1}^{n}\left\langle\Psi \mid \Psi_{\nu}\right\rangle\left\langle\Psi_{\nu}\right|$, where $\left\langle\Psi \mid \Psi_{\nu}\right\rangle=\int d \vec{\xi} \Psi(\vec{\xi}) f_{0}(\xi) \Psi_{\nu}(\vec{\xi})$ is the scalar product of two functions. $\left\{\Psi_{\nu}(\xi)\right\}$ is a set of functions satisfying the conditions $\left\langle\Psi_{\mu} \mid \Psi_{\nu}\right\rangle=\delta_{\mu \nu}, \sum_{\nu}\left|\Psi_{\nu}\right\rangle\left\langle\Psi_{\nu}\right|=1$. As we can see from the structure of elements of the matrixes $i \tilde{\Omega}^{G}(\vec{k})$ and $\tilde{\Pi}^{G}(\vec{k} ; z)$, the contributions of kinetic and potential parts of enthalpy are separated. However, all the transport kernels of $\tilde{\Pi}(\vec{k} ; z)$ are determined in terms of the time correlation functions (A.2), (A.4) and the transport kernels $\bar{\varphi}_{\dot{h} \dot{h}}^{(0)}\left(\vec{k} ; t, t^{\prime}\right), \bar{\varphi}_{j \dot{h}}^{(0)}\left(\vec{k} ; t, t^{\prime}\right)$, $\bar{\varphi}_{\dot{h} \jmath}^{(0)}\left(\vec{k} ; t, t^{\prime}\right)$ and $D\left(\vec{k} ; t, t^{\prime}\right)(\underline{3.16})$.

\section{TRANSITION TO EQUATIONS OF MOLECULAR HYDRODYNAMICS}

It is well known that the molecular hydrodynamics of dense gases and liquids is based on the transport equations for the averaged values of densities of particles number $\left\langle\hat{n}_{\vec{k}}\right\rangle^{t}$, momentum $\left\langle\hat{\vec{\jmath}}_{\vec{k}}\right\rangle^{t}$ and generalized enthalpy $\left\langle\hat{h}_{\vec{k}}\right\rangle^{t}\left[10\right.$, 48]. In our case $\left\langle\hat{h}_{\vec{k}}\right\rangle^{t}=\left\langle\hat{h}_{\vec{k}}^{k i n}\right\rangle^{t}+$ $\left\langle\hat{h}_{\vec{k}}^{\text {int }}\right\rangle^{t}$. As a result of projecting of the equations (3.19), (3.20) onto the first moments of the nonequilibrium one-particle distribution function we obtain a set of equations for the averages $\left\langle\hat{n}_{\vec{k}}\right\rangle^{t},\left\langle\hat{\vec{J}}_{\vec{k}}\right\rangle^{t},\left\langle\hat{h}_{\vec{k}}^{k i n}\right\rangle^{t}$ and $\left\langle\hat{h}_{\vec{k}}^{i n t}\right\rangle^{t}$ [with the elements of frequency matrix (33.27) and matrix of memory functions (3.28)], which in the Laplace representation we write down in the explicit form:

$$
\begin{aligned}
& z n_{\vec{k}}(z)+i \Omega_{n_{\jmath}}(\vec{k}) \vec{\jmath}_{\vec{k}}(z)=-\left\langle\hat{n}_{\vec{k}}(t=0)\right\rangle, \\
& z \vec{\jmath}_{\vec{k}}(z)+i \Omega_{\jmath n}(\vec{k}) n_{\vec{k}}(z)+i \Omega_{j h}^{k i n}(\vec{k}) h_{\vec{k}}^{k i n}(z)+i \Omega_{j h}^{i n t}(\vec{k}) h_{\vec{k}}^{i n t}(z) \\
& -\Pi_{\jmath \jmath}(\vec{k}, z) \vec{\jmath}_{\vec{k}}(z)-\Pi_{\jmath h}^{k i n}(\vec{k}, z) h_{\vec{k}}^{k i n}(z)-\Pi_{\jmath h}^{i n t}(\vec{k}, z) h_{\vec{k}}^{i n t}(z)=-\left\langle\hat{\vec{\jmath}}_{\vec{k}}(t=0)\right\rangle, \\
& z h_{\vec{k}}^{k i n}(z)+i \bar{\Omega}_{h \jmath}^{k i n}(\vec{k}, z) \vec{\jmath}_{\vec{k}}(z) \\
& -\Pi_{h h}^{k i n, k i n}(\vec{k}, z) h_{\vec{k}}^{k i n}(z)-\Pi_{h h}^{k i n, i n t}(\vec{k}, z) h_{\vec{k}}^{i n t}(z)=-\left\langle\hat{h}_{\vec{k}}^{k i n}(t=0)\right\rangle, \\
& z h_{\vec{k}}^{i n t}(z)+i \bar{\Omega}_{h \jmath}^{i n t}(\vec{k}, z) \vec{\jmath}_{\vec{k}}(z) \\
& -\prod_{h h}^{i n t, k i n}(\vec{k}, z) h_{\vec{k}}^{k i n}(z)-\prod_{h h}^{i n t, i n t}(\vec{k}, z) h_{\vec{k}}^{i n t}(z)=-\left\langle\hat{h}_{\vec{k}}^{i n t}(t=0)\right\rangle,
\end{aligned}
$$


where

$$
\begin{aligned}
& i \bar{\Omega}_{h \jmath}^{k i n}(\vec{k}, z)=i \Omega_{h \jmath}^{k i n}(\vec{k})-\Pi_{h \jmath}^{k i n}(\vec{k}, z), \\
& i \bar{\Omega}_{h \jmath}^{i n t}(\vec{k}, z)=i \Omega_{h \jmath}^{i n t}(\vec{k})-\Pi_{h \jmath}^{i n t}(\vec{k}, z) .
\end{aligned}
$$

In order to pass to the equations of molecular hydrodynamics let us sum up equations (4.3) and (4.4). As a result we obtain (superscript "tot" denotes the total enthalpy density):

$$
z h_{\vec{k}}(z)+i \bar{\Omega}_{h \jmath}(\vec{k}, z) \vec{\jmath}_{\vec{k}}(z)-\Pi_{h h}^{t o t, k i n}(\vec{k}, z) h_{\vec{k}}^{k i n}(z)-\Pi_{h h}^{t o t, i n t}(\vec{k}, z) h_{\vec{k}}^{i n t}(z)=\left\langle\hat{h}_{\vec{k}}(t=0)\right\rangle(4
$$

where

$$
\begin{aligned}
h_{\vec{k}}(z) & =h_{\vec{k}}^{k i n}(z)+h_{\vec{k}}^{i n t}(z), \\
i \bar{\Omega}_{h_{\jmath}}(\vec{k}, z) & =i \Omega_{h_{\jmath}}(\vec{k})-\Pi_{h \jmath}(\vec{k}, z) .
\end{aligned}
$$

Then, after some transformations we can preset this equation in the following form:

$$
\begin{aligned}
z h_{\vec{k}}(z) & +i \bar{\Omega}_{h \jmath}(\vec{k}, z) \vec{\jmath}_{\vec{k}}(z)-\Pi_{h h}(\vec{k}, z) h_{\vec{k}}(z) \\
& +\prod_{h h}^{t o t, k i n}(\vec{k}, z) h_{\vec{k}}^{i n t}(z)+\prod_{h h}^{t o t, i n t}(\vec{k}, z) h_{\vec{k}}^{k i n}(z)=-\left\langle\hat{h}_{\vec{k}}(t=0)\right\rangle,
\end{aligned}
$$

where

$$
\Pi_{h h}(\vec{k}, z)=\Pi_{h h}^{t o t, k i n}(\vec{k}, z)+\Pi_{h h}^{t o t, i n t}(\vec{k}, z) .
$$

To eliminate $h_{\vec{k}}^{i n t}(z)$ and $h_{\vec{k}}^{k i n}(z)$ from (4.9) we use equations (4.3), (4.4) once more, and find:

$$
\begin{aligned}
z h_{\vec{k}}^{i n t}(z) & +i \tilde{\Omega}_{h \jmath}^{i n t}(\vec{k}, z) \vec{\jmath}_{\vec{k}}(z)-\Sigma_{h h}^{i n t, i n t}(\vec{k}, z) h_{\vec{k}}^{i n t}(z)=0 \\
z h_{\vec{k}}^{k i n}(z) & +\left\{i \bar{\Omega}_{h \jmath}^{k i n}(\vec{k}, z)+\Pi_{h h}^{k i n, i n t}(\vec{k}, z)\left[z-\Sigma_{h h}^{i n t, i n t}(\vec{k}, z)\right]^{-1} i \tilde{\Omega}_{h \jmath}^{i n t}(\vec{k}, z)\right\} \vec{\jmath}_{\vec{k}}(z) \\
& -\prod_{h h}^{k i n, k i n}(\vec{k}, z) h_{\vec{k}}^{k i n}(z)=0
\end{aligned}
$$

where

$$
\begin{aligned}
i \tilde{\Omega}_{h \jmath}^{i n t}(\vec{k}, z) & =i \bar{\Omega}_{h \jmath}^{i n t}(\vec{k}, z)+\Pi_{h h}^{i n t, k i n}(\vec{k}, z)\left[z-\Pi_{h h}^{k i n, k i n}(\vec{k}, z)\right]^{-1} i \bar{\Omega}_{h \jmath}^{k i n}(\vec{k}, z), \\
\sum_{h h}^{i n t, i n t}(\vec{k}, z) & =\Pi_{h h}^{i n t, i n t}(\vec{k}, z)+\Pi_{h h}^{i n t, k i n}(\vec{k}, z)\left[z-\Pi_{h h}^{k i n, k i n}(\vec{k}, z)\right]^{-1} \Pi_{h h}^{k i n, i n t}(\vec{k}, z) .
\end{aligned}
$$

After expressing $h_{\vec{k}}^{i n t}(z)$ and $h_{\vec{k}}^{\text {kin }}(z)$ from (4.11), (4.12) we can present equation (4.9) as

$$
z h_{\vec{k}}(z)+\left(i \bar{\Omega}_{h_{\jmath}}(\vec{k}, z)-\Sigma_{h_{\jmath}}(\vec{k}, z)\right) \vec{\jmath}_{\vec{k}}(z)-\Pi_{h h}(\vec{k}, z) h_{\vec{k}}(z)=-\left\langle\hat{h}_{\vec{k}}(t=0)\right\rangle
$$


with

$$
\begin{aligned}
\Sigma_{h \jmath}(\vec{k}, z)= & \Pi_{h h}^{t o t, k i n}(\vec{k}, z) \frac{1}{z-\Sigma_{h h}^{i n t, i n t}(\vec{k}, z)} i \bar{\Omega}_{h \jmath}^{i n t}+\Pi_{h h}^{t o t, i n t}(\vec{k}, z) \frac{1}{z-\Pi_{h h}^{k i n, k i n}(\vec{k}, z)} \\
& \times\left(i \bar{\Omega}_{h \jmath}^{k i n}(\vec{k}, z)+\prod_{h h}^{k i n, i n t}(\vec{k}, z) \frac{1}{z-\sum_{h h}^{i n t, i n t}(\vec{k}, z)} i \tilde{\Omega}_{h \jmath}^{i n t}(\vec{k}, z)\right) .
\end{aligned}
$$

Now let us write down equation (4.2) in the following form:

$$
\begin{aligned}
z \vec{\jmath}_{\vec{k}}(z) & +i \Omega_{\jmath n}(\vec{k}) n_{\vec{k}}(z)+i \bar{\Omega}_{\jmath h}(\vec{k}, z) h_{\vec{k}}(z)-\Pi_{\jmath \jmath}(\vec{k}, z) \vec{\jmath}_{\vec{k}}(z) \\
& -i \bar{\Omega}_{\jmath h}^{i n t}(\vec{k}, z) h_{\vec{k}}^{k i n}(z)-i \bar{\Omega}_{\jmath h}^{i n t}(\vec{k}, z) h_{\vec{k}}^{i n t}(z)=-\left\langle\hat{\vec{\jmath}}_{\vec{k}}(t=0)\right\rangle,
\end{aligned}
$$

where

$$
\begin{aligned}
i \bar{\Omega}_{\jmath h}(\vec{k}, z) h_{\vec{k}}(z) & =i \Omega_{\jmath h}(\vec{k})-\Pi_{\jmath h}(\vec{k}, z), \\
i \bar{\Omega}_{\jmath h}^{i n t}(\vec{k}, z) h_{\vec{k}}(z) & =i \Omega_{\jmath h}^{i n t}(\vec{k})-\Pi_{\jmath h}^{i n t}(\vec{k}, z), \\
i \bar{\Omega}_{\jmath h}^{k i n}(\vec{k}, z) h_{\vec{k}}(z) & =i \Omega_{\jmath h}^{k i n}(\vec{k})-\Pi_{\jmath h}^{k i n}(\vec{k}, z) .
\end{aligned}
$$

After taking into account (4.11) and (4.12), we can present equation (4.9) in the form

$$
z \vec{\jmath}_{\vec{k}}(z)+i \Omega_{\jmath n}(\vec{k}) n_{\vec{k}}(z)+i \bar{\Omega}_{\jmath h}(\vec{k}, z) h_{\vec{k}}(z)-\Sigma_{\jmath \jmath}(\vec{k}, z) \vec{\jmath}_{\vec{k}}(z)=-\left\langle\hat{\vec{\jmath}}_{\vec{k}}(t=0)\right\rangle
$$

with

$$
\begin{aligned}
\Sigma_{\jmath \jmath}(\vec{k}, z)= & \Pi_{\jmath \jmath}(\vec{k}, z)-i \bar{\Omega}_{\jmath h}^{i n t}(\vec{k}, z) \frac{1}{z-\Pi_{h h}^{k i n, k i n}(\vec{k}, z)} \\
& \times\left\{i \bar{\Omega}_{h \jmath}^{k i n}(\vec{k}, z)+\Pi_{h h}^{k i n, k i n}(\vec{k}, z) \frac{1}{z-\sum_{h h}^{i n t, i n t}(\vec{k}, z)} i \tilde{\Omega}_{h \jmath}^{k i n}(\vec{k}, z)\right\} \\
& -i \bar{\Omega}_{\jmath h}^{k i n}(\vec{k}, z) \frac{1}{z-\Sigma_{h h}^{i n t, i n t}(\vec{k}, z)} i \tilde{\Omega}_{h \jmath}^{i n t}(\vec{k}, z) .
\end{aligned}
$$

Finally, the system of equation, which by its structure coincide with the set of equations of molecular hydrodynamics, can be presented as follows:

$$
\begin{aligned}
& z n_{\vec{k}}(z)+i \Omega_{n_{\jmath}}(\vec{k}) \vec{\jmath}_{\vec{k}}(z)=-\left\langle\hat{n}_{\vec{k}}(t=0)\right\rangle \\
& z \vec{\jmath}_{\vec{k}}(z)+i \Omega_{\jmath n}(\vec{k}) n_{\vec{k}}(z)+i \Omega_{\jmath h}(\vec{k}, z) h_{\vec{k}}(z) \\
&-\Pi_{\jmath h}(\vec{k}, z) h_{\vec{k}}(z)-\Sigma_{\jmath \jmath}(\vec{k}, z) \vec{\jmath}_{\vec{k}}(z)=-\left\langle\hat{\vec{\jmath}}_{\vec{k}}(t=0)\right\rangle, \\
& z h_{\vec{k}}(z)+i \Omega_{h_{\jmath}}(\vec{k}) \vec{\jmath}_{\vec{k}}(z)-\Pi_{h \jmath}(\vec{k}, z) \vec{\jmath}_{\vec{k}}(z)-\Pi_{h h}(\vec{k}, z) h_{\vec{k}}(z)=-\left\langle\hat{h}_{\vec{k}}(t=0)\right\rangle .
\end{aligned}
$$


Here, $\Sigma_{\jmath \jmath}(\vec{k}, z), \Pi_{h h}(\vec{k}, z), \Pi_{h \jmath}(\vec{k}, z)$ and $\Pi_{\jmath h}(\vec{k}, z)$ determine the generalized viscosity, heat conductivity coefficients as well as the generalized transport cross-coefficients describing dissipative correlations between viscous and heat processes.

It is worth to mention that the presented generalized transport kernels are expressed via the transport kernels $\varphi_{n n}\left(\vec{k} ; \vec{p}, \vec{p}^{\prime} ; t, t^{\prime}\right), \varphi_{n h}\left(\vec{k} ; \vec{p} ; t, t^{\prime}\right), \varphi_{h n}\left(\vec{k} ; \vec{p}^{\prime} ; t, t^{\prime}\right)$ and $\varphi_{h h}\left(\vec{k} ; t, t^{\prime}\right)$ of a consistent description of kinetics and hydrodynamics and, obviously, their calculation depends on an explicit model of interparticle interaction.

\section{SPECTRUM OF COLLECTIVE EXCITATIONS}

Let us consider the system of kinetic equations (2.8), (2.9) in the case when the potential of interaction is presented as follows:

$$
\Phi\left(\left|\vec{r}_{i j}\right|\right)=\Phi^{\mathrm{hs}}\left(\left|\vec{r}_{i j}\right|\right)+\Phi^{\mathrm{l}}\left(\left|\vec{r}_{i j}\right|\right)
$$

where $\Phi^{\mathrm{hs}}\left(\left|\vec{r}_{i j}\right|\right)$ is the hard sphere interaction potential, and $\Phi^{\mathrm{l}}\left(\left|\vec{r}_{i j}\right|\right)$ is the long-range potential. Taking into account the features of the hard sphere model dynamics [12] and the results of investigations [31, 36, 42], one can separate Enskog-Boltzmann collision integral from the function $\varphi_{n n}\left(\vec{k} ; \vec{p}, \vec{p}^{\prime} ; t, t^{\prime}\right)$. Indeed, an infinitesimal time of a collision $\tau_{0} \rightarrow+0$ within an infinitesimal region $\sigma \pm \Delta r_{0}, \Delta r_{0} \sim\left|\tau_{0}\right|\left|\vec{p}_{2}-\vec{p}_{1}\right| / m \rightarrow+0$ being a feature of the hard sphere model dynamics ( $\sigma$ is the hard sphere diameter). Taking this into account in the kinetic equation (2.8) we can obtain the kinetic equation of the revised Enskog theory for the hard sphere model and the kinetic Enskog-Landau equation for the charged hard sphere model in a pair collision approximation, respectively [12]. In the latter case, when $\Phi^{\mathrm{l}}\left(\left|\vec{r}_{i j}\right|\right)$ is the Coulomb potential of interaction, taking into account the features $\tau_{0} \rightarrow+0$, $\Delta r_{0} \rightarrow+0$ makes it possible to separate a collision integral of the revised Enskog theory and a Landau-like collision integral in the limits $\tau \rightarrow-0$ and $\tau \rightarrow-\infty$, respectively. In the case of potential (5.1), in the region of $\tau_{0} \rightarrow+0, \Delta r_{0} \rightarrow+0, \sigma \pm \Delta r_{0}$ when the main contribution to a dynamics is defined by pair collisions of hard spheres, the memory func-

tion $\varphi_{n n}\left(\vec{k} ; \vec{p}, \vec{p}^{\prime} ; t, t^{\prime}\right)$ can be calculated by expanding it over the density (a pair collision approximation), what was done in papers by Mazenko [36-39, 42] in detail. 
Then, the kinetic equation (2.8) can be represented in the following form:

$$
\begin{aligned}
& \frac{\partial}{\partial t} f_{\vec{k}}(\vec{p} ; t)+\frac{i \vec{k} \cdot \vec{p}}{m} f_{\vec{k}}(\vec{p} ; t)=-\frac{i \vec{k} \cdot \vec{p}}{m} n f_{0}(\vec{p}) c_{2}(k) \int d \vec{p}^{\prime} f_{\vec{k}}\left(\vec{p}^{\prime} ; t\right) \\
& \quad-\int d \vec{p}^{\prime} \varphi_{n n}^{\mathrm{hs}}\left(\vec{k}, \vec{p}, \vec{p}^{\prime}\right) f_{\vec{k}}\left(\vec{p}^{\prime} ; t\right)+i \Omega_{n h}(\vec{k} ; \vec{p}) h_{\vec{k}}^{\mathrm{int}}(t) \\
& \quad-\int d \vec{p}^{\prime} \int_{-\infty}^{t} d t^{\prime} e^{\varepsilon\left(t-t^{\prime}\right)} \varphi_{n n}^{1}\left(\vec{k} ; \vec{p}, \vec{p}^{\prime} ; t, t^{\prime}\right) f_{\vec{k}}\left(\vec{p}^{\prime} ; t^{\prime}\right)-\int_{-\infty}^{t} d t^{\prime} e^{\varepsilon\left(t-t^{\prime}\right)} \varphi_{n h}\left(\vec{k} ; \vec{p} ; t, t^{\prime}\right) h_{\vec{k}}^{\mathrm{int}}\left(t^{\prime}\right) .
\end{aligned}
$$

Here,

$$
\begin{aligned}
& \int d \vec{p}^{\prime} \varphi_{n n}^{\mathrm{hs}}\left(\vec{k}, \vec{p}, \vec{p}^{\prime}\right) f_{\vec{k}}\left(\vec{p}^{\prime} ; t\right)=n g_{2}(\sigma) \sigma^{2} \int d \Omega_{\sigma} \int d \vec{p}^{\prime} \frac{\left(\vec{p}-\vec{p}^{\prime}\right) \cdot \hat{\vec{\sigma}}}{m} \Theta_{-}\left(\hat{\vec{\sigma}} \cdot\left[\vec{p}-\vec{p}^{\prime}\right]\right) \\
& \times\left[f_{0}\left(p^{\prime *}\right) f_{\vec{k}}(\vec{p} ; t)-f_{0}\left(p^{\prime}\right) f_{\vec{k}}\left(\vec{p}^{*} ; t\right)+e^{i \vec{k} \cdot \hat{\vec{\sigma}} \sigma} f_{0}\left(p^{\prime *}\right) f_{\vec{k}}\left(\vec{p}^{*} ; t\right)-e^{i \vec{k} \cdot \hat{\vec{\sigma}} \sigma} f_{0}(p) f_{\vec{k}}\left(\vec{p}^{\prime} ; t\right)\right]
\end{aligned}
$$

is the Enskog-Boltzmann collision integral, where $c_{2}^{0}(\vec{k})$ is the low-density limit of the direct correlation function and $g_{2}(\sigma)$ is the pair distribution function. The step function $\Theta_{-}(x)$ is unity for $x<0$ and vanishes otherwise. $d \Omega_{\sigma}$ is the differential solid angle, $\hat{\vec{\sigma}}$ is unity vector. The pre- and postcollision momenta of the colliding hard spheres are denoted as $\left(\vec{p}, \vec{p}^{\prime}\right)$ and $\left(\vec{p}^{*}, \vec{p}^{*}\right)$, respectively. $\varphi_{n n}^{1}\left(\vec{k} ; \vec{p}, \vec{p}^{\prime} ; t, t^{\prime}\right)$ is the part of the transport kernel related to the long-range interaction potential $\Phi^{\mathrm{l}}\left(\left|\vec{r}_{i j}\right|\right)$. Notably, the presented equation contains the Enskog-Boltzmann collision integral describing short-time dynamics of the hard sphere model. The collective effects related to the long-range interactions between particles are described by the functions $i \Omega_{n h}(\vec{k} ; \vec{p}), \varphi_{n n}^{\mathrm{l}}\left(\vec{k} ; \vec{p}, \vec{p}^{\prime} ; t, t^{\prime}\right), \varphi_{n h}\left(\vec{k} ; t, t^{\prime}\right)$ and by the equation for $h_{\vec{k}}^{\text {int }}(t)$. Since the collective modes for the Enskog-Boltzmann model are well studied [31], the investigation of time correlation functions and collective modes for the system of particles interacting through the potential (5.1) turns out to be of great interest. In the case of the hard sphere system, the set of kinetic equations (2.9), (5.2) reduces to the Enskog-Boltzmann kinetic equation [31].

$$
\begin{aligned}
& \frac{\partial}{\partial t} f_{\vec{k}}(\vec{p} ; t)+\frac{i \vec{k} \cdot \vec{p}}{m} f_{\vec{k}}(\vec{p} ; t)=-\frac{i \vec{k} \cdot \vec{p}}{m} n f_{0}(\vec{p})\left[c_{2}(k)-g_{2}(\sigma) c_{2}^{0}(k)\right] \int d \vec{p}^{\prime} f_{\vec{k}}\left(\vec{p}^{\prime} ; t\right) \\
& \quad-n g_{2}(\sigma) \sigma^{2} \int d \Omega_{\sigma} \int d \vec{p}^{\prime} \frac{\left(\vec{p}-\vec{p}^{\prime}\right) \cdot \hat{\vec{\sigma}}}{m} \Theta_{-}\left(\hat{\vec{\sigma}} \cdot\left[\vec{p}-\vec{p}^{\prime}\right]\right) \\
& \quad \times\left[f_{0}\left(p^{\prime *}\right) f_{\vec{k}}(\vec{p} ; t)-f_{0}\left(p^{\prime}\right) f_{\vec{k}}\left(\vec{p}^{*} ; t\right)+e^{i \vec{k} \cdot \hat{\vec{\sigma} \sigma}} f_{0}\left(p^{\prime *}\right) f_{\vec{k}}\left(\vec{p}^{*} ; t\right)-e^{i \vec{k} \cdot \hat{\vec{\sigma} \sigma}} f_{0}(p) f_{\vec{k}}\left(\vec{p}^{\prime} ; t\right)\right] .
\end{aligned}
$$

Projecting the Enskog-Boltzmann equation onto the first moments of the nonequilibrium one-particle distribution function a spectrum of collective excitations for the hard sphere 
model was obtained in [31, 49]. Herewith, it is important to note that for the kinetic Enskog-Boltzmann equation we can consider two typical limits: $k \sigma \ll 1$ and $k \sigma \gg 1$. In the case when $k \sigma \ll 1$ the spectrum includes: heat mode $z_{\mathrm{H}}(k)=-D_{\mathrm{TE}} k^{2}$, where $D_{\mathrm{TE}}$ is the thermal diffusivity coefficient in the Enskog transport theory [50]; two sound modes with eigenvalues given by $z_{ \pm}(k)= \pm i c k-\Gamma_{\mathrm{E}} k^{2}$, where $\Gamma_{\mathrm{E}}$ is the sound damping coefficient and $c$ is the sound velocity in the Enskog theory; two transverse modes with eigenvalues given by $z_{\nu_{1}}(k)=z_{\nu_{2}}(k)=z_{\nu}(k)=-\nu_{\mathrm{E}} k^{2}, \nu_{\mathrm{E}}$ is the kinematic viscosity in the Enskog dense gas theory. In the limit when $k \sigma \gg 1$ the Enskog-Boltzmann collision integral (5.3) is transformed [31] into the Lorentz-Boltzmann collision integral which has only one eigenfunction $\Psi_{1}(\vec{p})=1$. Consequently, we obtain the diffusion mode only with the eigenvalue $z_{\mathrm{D}}(k)=-D_{\mathrm{E}} k^{2}, D_{\mathrm{E}}$ is the self-diffusion coefficient as given by the Enskog dense gas theory.

Let us now project the system of equation (2.9), (5.2) onto the first moments of the nonequilibrium one-particle distribution function. Thereafter we perform a simple transformations consisting in the transition from the set of equations (3.25) for averages $n_{\vec{k}}(z), \vec{\jmath}_{\vec{k}}(z), h_{\vec{k}}^{\text {kin }}(z), h_{\vec{k}}^{\text {int }}(z)$ to the equations of the generalized hydrodynamics for averages $\tilde{b}_{\vec{k}}(z)=\left[n_{\vec{k}}(z), \vec{\jmath}_{\vec{k}}(z), h_{\vec{k}}(z)=h_{\vec{k}}^{\text {kin }}(z)+h_{\vec{k}}^{\text {int }}(z)\right]$ as it was described in section IV] This permits to correctly define (see below) the generalized viscosity coefficient via the transport kernel (4.22) and the heat conductivity coefficient via the transport kernel $\Pi_{h h}(\vec{k}, z)$. The averages $\tilde{b}_{\vec{k}}(z)$ satisfy the set of equations

$$
z \tilde{b}_{\vec{k}}(z)-\tilde{\Sigma}^{\mathrm{H}}(\vec{k} ; z) \tilde{b}_{\vec{k}}(z)=-\left\langle\tilde{b}_{\vec{k}}(t=0)\right\rangle^{t} .
$$

In the limit $k \sigma \gg 1$ the latter reduces to the single equation of diffusion for $n_{\vec{k}}(z)$ in which the transport kernel $\Sigma^{\mathrm{H}}(\vec{k} ; z)=\left\langle\Psi_{1}\left|\varphi_{n n}^{\mathrm{L}-\mathrm{B}}(\vec{k})\right| \Psi_{1}\right\rangle$ corresponds to the Lorentz-Boltzmann collision integral (5.3). In the opposite case, when $k \sigma \ll 1$, matrix $\tilde{\Sigma}^{\mathrm{H}}(\vec{k} ; z)$ is defined as follows: $\tilde{\Sigma}^{\mathrm{H}}(\vec{k} ; z)=i \tilde{\Omega}^{\mathrm{H}}(\vec{k})-\tilde{\Pi}^{\mathrm{H}}(\vec{k} ; z)$,

$$
\tilde{\Sigma}_{\mathrm{H}}(\vec{k} ; z)=\left(\begin{array}{lll}
0 & i \Omega_{n \jmath} & 0 \\
i \Omega_{\jmath n} & -\left\langle\Psi_{2}\left|\varphi_{n n}^{\mathrm{hs}}\right| \Psi_{2}\right\rangle-\Sigma_{j j}^{\mathrm{l}} & i \Omega_{\jmath h}-\Pi_{\jmath h} \\
0 & i \Omega_{h \jmath}-\Pi_{h \jmath} & -\left\langle\Psi_{3}\left|\varphi_{n n}^{\mathrm{hs}}\right| \Psi_{3}\right\rangle-\Pi_{h h}^{\mathrm{l}}
\end{array}\right)_{(\vec{k}, z)}
$$

with $\Sigma_{j j}(\vec{k}, z)$ defined by (4.22) and

$$
\Pi_{h h}(\vec{k}, z)=\Pi_{h h}^{\mathrm{kin}, \mathrm{kin}}(\vec{k}, z)+\Pi_{h h}^{\mathrm{kin}, \mathrm{int}}(\vec{k}, z)+\Pi_{h h}^{\mathrm{int}, \mathrm{kin}}(\vec{k}, z)+\Pi_{h h}^{\mathrm{int}, \mathrm{int}}(\vec{k}, z)
$$


which is equivalent to (4.10).

We can separate real and imaginary parts in memory functions (4.22) and (5.7) as follows:

$$
\Sigma_{j j}(\vec{k}, z)=\Sigma_{j j}^{\prime}(\vec{k}, \omega)+i \Sigma_{j j}^{\prime \prime}(\vec{k}, \omega), \quad \Pi_{h h}(\vec{k}, z)=\Pi_{h h}^{\prime}(\vec{k}, \omega)+i \Pi_{h h}^{\prime \prime}(\vec{k}, \omega)
$$

Herewith, the contributions from the hard sphere dynamics with typical spatial-temporal scale $\tau_{0} \rightarrow+0, \Delta r_{0} \rightarrow+0$ are separated in the transport kernel $\varphi_{n n}\left(\vec{k} ; \vec{p}, \vec{p}^{\prime} ; t, t^{\prime}\right)$ only in the first term in the right-hand side of elements (3.29) and hence in (4.22). After these transformations we can obtain a spectrum of collective excitations in the hydrodynamic limit $k \sigma \ll 1$. Heat mode is defined as follows:

$$
z_{\mathrm{H}}(k)=-D_{\mathrm{T}} k^{2}, \quad D_{\mathrm{T}}=D_{\mathrm{TE}}+D_{\mathrm{T}}^{\mathrm{l}},
$$

where $D_{\mathrm{T}}$ is the thermal diffusivity coefficient for the system with the potential of interaction (5.1). In (5.9) $D_{\mathrm{T}}^{1}$ is determined through the corresponding elements (3.29) of matrix of transport kernels (3.28):

$$
D_{\mathrm{T}}^{1}=\frac{\lambda^{\mathrm{l}}}{n m c_{p}}, \quad \lambda^{1}=\lim _{\vec{k} \rightarrow 0, \omega \rightarrow 0} \lambda^{\mathrm{l}}(\vec{k}, \omega), \quad \lambda^{\mathrm{l}}(\vec{k}, \omega)=\frac{c_{v}(k)}{k_{\mathrm{B}} \beta^{2}} \frac{1}{k^{2}} \Pi_{h h}^{\prime \prime}(\vec{k}, \omega) .
$$

Here, $\lambda^{1}$ is a hydrodynamic limiting value of the generalized heat conductivity coefficient $\lambda^{1}(\vec{k}, \omega)$ defined via elements of the matrix (3.28). $c_{p}$ and $c_{v}$ are the $\vec{k} \rightarrow 0$ limit values of the generalized heat capacity at constant pressure $c_{p}(k)$ and at constant volume $c_{v}(k)$, respectively. Two sound modes

$$
z_{ \pm}(k)= \pm i c k-\Gamma k^{2}, \quad c=\frac{\gamma}{\beta m S(0)}, \quad \Gamma=\frac{1}{2}\left[(\gamma-1) D_{\mathrm{T}}+\eta^{\mathrm{L}}\right],
$$

where $c$ is the sound velocity in the system with the potential of interaction (5.1), $\gamma=c_{p} / c_{v}$, and $S(0)$ is a limiting value of a static structure factor $S(k)$ of the system with potential (5.1). $\Gamma$ is the sound damping coefficient.

$$
\eta^{\mathrm{L}}=\left(\frac{4}{3} \eta^{\perp}+\eta^{\mathrm{b}}\right) / m n, \quad \eta^{\mathrm{b}}=\eta_{\mathrm{E}}^{\mathrm{b}}+\eta_{\mathrm{l}}^{\mathrm{b}}, \quad \eta^{\perp}=\eta_{\mathrm{E}}^{\perp}+\eta_{\mathrm{l}}^{\perp}
$$

$\eta^{\mathrm{L}}$ is the longitudinal viscosity defined via the bulk viscosity $\eta^{\mathrm{b}}$ and the shear viscosity $\eta^{\perp}$ coefficients, respectively. $\eta_{\mathrm{E}}^{\perp}$ is the shear viscosity in Enskog theory, and $\eta_{1}^{\perp}$ is the value of the generalized shear viscosity coefficient $\eta_{1}^{\perp}(\vec{k}, \omega)$ [defined via elements of the matrix (3.28) ] calculated in the hydrodynamic limit

$$
\eta_{\mathrm{l}}^{\perp}=\lim _{\vec{k} \rightarrow 0, \omega \rightarrow 0} \eta_{\mathrm{l}}^{\perp}(\vec{k}, \omega), \quad \eta_{\mathrm{l}}^{\perp}(\vec{k}, \omega)=\frac{m n}{\beta} \frac{1}{k^{2}} \Sigma_{j j}^{\prime \prime \perp}(\vec{k}, \omega) .
$$


$\Sigma_{j j}^{\perp}(\vec{k}, z)$ is the transverse component of the generalized transport kernel $\Sigma_{j j}(\vec{k}, z)$, where the wave vector $\vec{k}$ is directed along the $0 Z$ axis. The longitudinal viscosity coefficient $\eta_{1}^{\mathrm{L}}$ is calculated in the hydrodynamic limit

$$
\eta_{1}^{\mathrm{L}}=\lim _{\vec{k} \rightarrow 0, \omega \rightarrow 0} \eta_{1}^{\mathrm{L}}(\vec{k}, \omega), \quad \eta_{1}^{\mathrm{L}}(\vec{k}, \omega)=\frac{m n}{\beta} \frac{1}{k^{2}} \sum_{j j}^{\prime \prime \mathrm{L}}(\vec{k}, \omega),
$$

with $\eta_{1}^{\mathrm{L}}(\vec{k}, \omega)$ being the generalized longitudinal viscosity coefficient defined via longitudinal components of the generalized transport kernel $\Sigma_{j j}(\vec{k}, z)$. Two transverse modes with the eigenvalues given by

$$
z_{\nu}(k)=-\nu k^{2}, \quad \nu=\frac{\eta \frac{\perp}{\mathrm{E}}+\eta_{1}^{\perp}}{n m} .
$$

$\nu$ is the kinematic viscosity for the system with the potential of interaction (5.1).

In the limit $k \sigma \gg 1$, we obtain a diffusion mode, with the eigenvalue

$$
z_{\mathrm{D}}(k)=-D_{\mathrm{E}} k^{2}
$$

which is the same as in the Enskog theory.

As we can see from the above expressions, presence of the long-range part in the potential of interaction entails a renormalization of all the damping coefficients in the collective modes spectrum. In particular, contributions related to long-range potential appear in heat and sound modes as well as in transverse modes. Nevertheless, diffusion mode remains unchanged.

When obtaining the spectrum of collective excitations based on the transport equations (5.2), (2.9) in the limit $|\vec{k}| \rightarrow 0, \omega \rightarrow 0$ we have not specificated the form of the long-range potential of interaction $\Phi^{1}\left(\left|\vec{r}_{i j}\right|\right)$. Obviously, for every $|\vec{k}|$ and $\omega$ from the whole range of their values, the contributions from short-range and long-range parts of potential of interaction into kinetic and hydrodynamic processes will be different and it is difficult to solve this problem in general. The kinetic processes connected with the particles scattering will be affected by both short- and long-range parts of interaction potential. Obviously, the long-wavelength hydrodynamic processes will be affected by the long-range part of potential $\Phi^{1}\left(\left|\vec{r}_{i j}\right|\right)$ sufficiently, in particular, when the latter can be presented via the Fourier-components of the particles number density (3.22). In order to evaluate the contribution of the long-range part into the collision integral $\int d \vec{p}^{\prime} \int_{-\infty}^{t} d t^{\prime} e^{\varepsilon\left(t-t^{\prime}\right)} \varphi_{n n}^{1}\left(\vec{k} ; \vec{p}, \vec{p}^{\prime} ; t, t^{\prime}\right) f_{\vec{k}}\left(\vec{p}^{\prime} ; t^{\prime}\right)$ we can employ an 
approach used to obtain the Enskog-Landau kinetic equation [12] in the second order in long-range interaction. For our case of weakly nonequilibrium processes, we obtain:

$$
\begin{aligned}
& \int d \vec{p}^{\prime} \int_{-\infty}^{t} d t^{\prime} e^{\varepsilon\left(t-t^{\prime}\right)} \varphi_{n n}^{1}\left(\vec{k} ; \vec{p}, \vec{p}^{\prime} ; t, t^{\prime}\right) f_{\vec{k}}\left(\vec{p}^{\prime} ; t^{\prime}\right)= \\
& -n \vec{F}(\vec{k} ; t) \cdot \frac{\partial}{\partial \vec{p}} f_{\vec{k}}(\vec{p} ; t)+n \int d \vec{v}^{\prime} \int d \varphi \int_{0}^{\infty} d b|\vec{q}| b\left[f_{0}\left(\vec{v}^{* *}\right) f_{\vec{k}}(\vec{v} ; t)-f_{0}(\vec{v}) f_{\vec{k}}\left(\vec{v}^{*} ; t\right)\right]
\end{aligned}
$$

where $\vec{F}(\vec{k} ; t)$ is the Fourier transform of the function $\vec{F}(r ; t)=\frac{\partial}{\partial r} \Phi^{1}(r) g_{2}(r ; t), b$ is the impact parameter, $\varphi$ is the azimuthal angle of scattering. $\vec{v}^{*}, \vec{v}^{*}$ are the particle velocities after collision:

$$
\begin{gathered}
\vec{v}^{*}=\vec{v}+\Delta \vec{v}, \quad \vec{v}^{*}=\vec{v}^{\prime}+\Delta \vec{v}, \quad \Delta \vec{g}^{*}=\vec{g}^{*}-\vec{g}=2 \Delta \vec{v}, \quad \vec{g}^{*}=\vec{v}^{*}-\vec{v}^{*}, \\
\Delta \vec{g}^{*}=\left.\frac{1}{\mu^{*}|\vec{g}|} \int^{\infty-\infty} d \xi\left(\frac{\partial}{\partial \vec{r}} \Phi^{1}(r)\right) g_{2}(r ; t)\right|_{r=\sqrt{b^{2}-\xi^{2}}},
\end{gathered}
$$

$\xi$ is the distance along centreline of cylinder of two particles scattering, $\mu^{*}$ is the reduced mass and $g_{2}(r ; t)$ is the two-particle quasiequilibrium coordinate distribution function. Herewith, it is assumed that scattering angles of particles a small, namely, $\chi^{*}(b, \vec{g})=\left|\Delta \vec{g}^{*}\right| /|\vec{g}| \ll 1$. Thereby, it is possible to take into account an effect of the long-range part of the interaction potential in the region of particles scattering with a transfer of momentum.

Another important contribution of long-range part of interaction potential $\Phi^{1}\left(\left|\vec{r}_{i j}\right|\right)$ is connected with transport kernels (3.15)-(3.16), the potential part of the generalized heat conductivity coefficient $\bar{\varphi}_{\dot{h} \dot{h}}^{(0)}\left(\vec{k} ; t, t^{\prime}\right)$ as well as the coefficients $\bar{\varphi}_{j \dot{h}}^{(0)}\left(\vec{k} ; t, t^{\prime}\right)$ and $\bar{\varphi}_{\dot{h}_{\jmath}}\left(\vec{k} ; t, t^{\prime}\right)$ describing cross-correlations between viscous and heat processes. In particular, to calculate $\bar{\varphi}_{\dot{h} \dot{h}}^{(0)}\left(\vec{k} ; t, t^{\prime}\right)$ it is necessary to reveal the action of Liouville operator onto $\hat{h}_{\vec{k}}^{\text {int }}$. Taking into account (3.22) with contribution from short-range interaction we obtain

$$
\begin{aligned}
\dot{\hat{h}}_{\vec{k}}^{i n t}= & -i \vec{k} \cdot \sum_{i \neq j} \frac{\vec{p}_{i}}{m} \Phi^{\mathrm{sh}}\left(\left|\vec{r}_{i j}\right|\right) e^{-i \vec{k} \cdot \vec{r}_{i}} \\
& +\frac{1}{2 m} \sum_{\vec{q}} \nu(q)\left[-i(\vec{q}+\vec{k}) \cdot \hat{\vec{j}}_{\vec{q}+\vec{k}} \hat{n}_{-\vec{q}}+i \vec{q} \cdot \hat{n}_{\vec{q}+\vec{k}} \hat{\vec{j}}_{-\vec{q}}\right] \\
& -\sum_{\vec{q}} \nu(q) S_{3}(\vec{q}+\vec{k},-\vec{q},-\vec{k}) S_{2}^{-1}(\vec{q}) i \vec{k} \cdot \hat{\vec{j}}_{-\vec{k}} .
\end{aligned}
$$

Taking into account the structure of $\dot{\hat{h}}_{\vec{k}}^{i n t}$ for $\bar{\varphi}_{\dot{h} \dot{h}}^{(0)}\left(\vec{k} ; t, t^{\prime}\right)$ we obtain:

$$
\bar{\varphi}_{\dot{h} \dot{h}}^{(0)}\left(\vec{k} ; t, t^{\prime}\right)=\bar{\varphi}_{\dot{h} \dot{h}}^{s h, s h}\left(\vec{k} ; t, t^{\prime}\right)+\bar{\varphi}_{\dot{h} \dot{h}}^{s h, l}\left(\vec{k} ; t, t^{\prime}\right)+\bar{\varphi}_{n j j}^{l l}\left(\vec{k} ; t, t^{\prime}\right)+\bar{\varphi}_{n j n j}^{l l}\left(\vec{k} ; t, t^{\prime}\right),
$$


where, in particular,

$$
\begin{aligned}
& \bar{\varphi}_{\dot{h} \dot{h}}^{s h, s h}\left(\vec{k} ; t, t^{\prime}\right)=k^{2}\left\langle\sum_{i j} \frac{\vec{p}_{i}}{m} \Phi^{\mathrm{sh}}\left(\left|\vec{r}_{i j}\right|\right) e^{-\vec{k} \vec{r}_{i}} T_{0}\left(t, t^{\prime}\right) \sum_{l j} \frac{\vec{p}_{l}}{m} \Phi^{\mathrm{sh}}\left(\left|\vec{r}_{l j}\right|\right) e^{\vec{k} \vec{r}_{l}}\right\rangle_{0} \Phi_{h h}^{-1}(\vec{k}) \\
& \bar{\varphi}_{n j n j}^{l l}\left(\vec{k} ; t, t^{\prime}\right)=\left(\frac{1}{2 m}\right)^{2} \sum_{\vec{q} \vec{q}^{\prime}} \nu(q) \nu\left(q^{\prime}\right) \\
& \times\left[-(\vec{q}+\vec{k}) \cdot\left\langle\hat{\vec{j}}_{\vec{q}+\vec{k}} \hat{n}_{-\vec{q}} T_{0}\left(t, t^{\prime}\right) \hat{\vec{j}}_{\vec{q}^{\prime}-\vec{k}} \hat{n}_{-\vec{q}^{\prime}}\right\rangle_{0} \cdot\left(\vec{q}^{\prime}-\vec{k}\right)+(\vec{q}+\vec{k}) \cdot\left\langle\hat{\vec{j}}_{\vec{q}+\vec{k}} \hat{n}_{-\vec{q}} T_{0}\left(t, t^{\prime}\right) \hat{n}_{-\vec{q}^{\prime}-\vec{k}} \hat{\vec{j}}_{-\vec{q}^{\prime}}\right\rangle_{0} \cdot \vec{q}^{\prime}\right. \\
& \left.+\vec{q} \cdot\left\langle\hat{n}_{\vec{q}+\vec{k}} \hat{\vec{j}}_{-\vec{q}} T_{0}\left(t, t^{\prime}\right) \hat{\vec{j}}_{\vec{q}^{\prime}-\vec{k}} \hat{n}_{-\vec{q}^{\prime}}\right\rangle_{0} \cdot\left(\vec{q}^{\prime}-\vec{k}\right)-\vec{q} \cdot\left\langle\hat{\vec{n}}_{\vec{q}+\vec{k}} \hat{j}_{-\vec{q}} T_{0}\left(t, t^{\prime}\right) \hat{n}_{-\vec{q}^{\prime}-\vec{k}} \hat{\vec{j}}_{-\vec{q}^{\prime}}\right\rangle_{0} \cdot \vec{q}^{\prime}\right] \Phi_{h h}^{-1}(\vec{k})
\end{aligned}
$$

Here, the time correlation function $\bar{\varphi}_{\dot{h} \dot{h}}^{s h, s h}\left(\vec{k} ; t, t^{\prime}\right)$ contributes from the short-range interaction and depends on the model for potential $\Phi^{\mathrm{sh}}\left(\left|\vec{r}_{i j}\right|\right) \cdot \bar{\varphi}_{\dot{h} \dot{h}}^{s h}\left(\vec{k} ; t, t^{\prime}\right)$ is the time correlation function describing cross-correlations between contributions from the short- and long-range parts of the potential of interaction. $\bar{\varphi}_{n j j}^{l l}\left(\vec{k} ; t, t^{\prime}\right)$ and $\bar{\varphi}_{n j n j}^{l l}\left(\vec{k} ; t, t^{\prime}\right)$ are the time correlation functions of the third and the fourth order in dynamic variables $\hat{\vec{j}}, \hat{n} \hat{\vec{j}}$ with the evolution operator $T_{0}\left(t, t^{\prime}\right)$. Investigating the time correlation functions for $\bar{\varphi}_{n j n j}^{l l}\left(\vec{k} ; t, t^{\prime}\right)$ an approximation of the mode coupling type [3] via the time correlation functions $\Phi_{n n} \Phi_{j j}+\Phi_{n j} \Phi_{j n}$ can be used. This issue needs a more detailed investigation within a certain model for the potential of interaction between particles.

\section{CONCLUSIONS}

In summary, in the present paper we consider the set of equations for the nonequilibrium one-particle distribution function and the potential part of the enthalpy density obtained within the framework of a consistent description of kinetics and hydrodynamics in dense gases and liquids. In this set of equations the collision integral of the kinetic equation has the Fokker-Planck form with the generalized friction coefficient in momentum space. It was shown that in this approach the viscosity processes are described in terms of the generalized diffusion and friction coefficients in the phase space. Applying the procedure of projecting onto the moments of the nonequilibrium distribution function to the equations (2.9), (5.2) we obtain a set of equations for extended set of variables $\left\{n_{\vec{k}}(z), \vec{\jmath}_{\vec{k}}(z), h_{\vec{k}}^{\text {kin }}(z), h_{\vec{k}}^{i n t}(z)\right\}$ and perform the transition to the transport equations of molecular hydrodynamics.

Within the framework of consistent description of kinetic and hydrodynamic processes we considered a set of kinetic equations for the potential of interaction of the system presented 
by the sum of hard spheres potential $\Phi^{\mathrm{hs}}\left(\left|\vec{r}_{i j}\right|\right)$ and a certain smooth one $\Phi^{\mathrm{l}}\left(\left|\vec{r}_{i j}\right|\right)$. In this case, we separated the Enskog-Boltzmann collision integral describing a collision dynamics at short distances from the collision integral of the kinetic equation for the nonequilibrium distribution function. Applying the procedure of projecting and passing to equations of molecular hydrodynamics we obtain a set of equations which allow us to investigate a spectrum of collective excitations in the limits $k \sigma \ll 1$ and $k \sigma \gg 1$. We showed that, besides the contribution from the hard spheres potential, all hydrodynamic modes contain contributions from the long-range part of potential. These contributions make the damping coefficients closer to the ones known from the hydrodynamic theory. Here, we formally presented the contribution from the long-ranged part of potential, since the latter, for example the Coulomb one, will contribute into the transport kernels (2.11). Moreover, we can separate the linearized Landau-like collision integral (5.17) describing pair collisions in

$\varphi_{n n}\left(\vec{k} ; \vec{p}, \vec{p}^{\prime} ; t, t^{\prime}\right)$, while $\varphi_{h n}\left(\vec{k} ; \vec{p} ; t, t^{\prime}\right), \varphi_{n h}\left(\vec{k} ; \vec{p} ; t, t^{\prime}\right), \varphi_{h h}\left(\vec{k} ; \vec{p} ; t, t^{\prime}\right)$ take into account collective Coulombic interactions. Evidently, calculation of the elements (3.29) of matrix $\tilde{\Pi}(\vec{k} ; z)$ will depend on the model of time dependence (exponential, Gaussian etc.) for transport kernels (2.11). When a spectrum of collective excitations is known, a whole set of time correlation functions can be investigated. In particular, it makes possible to investigate the behaviour of the dynamic structure factor and, in the case of potential (5.1), to separate a contributions from the hard spheres potential and the long-range part of potential in it.

\section{Appendix}

Here we present some correlation functions appearing in section III. Correlation functions $\bar{\varphi}_{n n}^{(1)}\left(\vec{k} ; \vec{p}, \vec{p}^{\prime} ; t, t^{\prime}\right)$ entering the equations (3.9) has the following form:

$$
\begin{aligned}
& \bar{\varphi}_{n n}^{(1)}\left(\vec{k} ; \vec{p}, \vec{p}^{\prime} ; t, t^{\prime}\right)=\frac{\beta}{m} \vec{p} \cdot \vec{\Phi}_{F h}(\vec{k}) f_{0}(p) \varphi_{h \jmath}\left(\vec{k} ; \vec{p}^{\prime} ; t, t^{\prime}\right) \cdot \frac{i \vec{k}}{m} \\
& \quad+-\frac{\vec{k}}{m} \cdot \vec{p} \varphi_{n \jmath}\left(\vec{k} ; \vec{p}, \vec{p}^{\prime} ; t, t^{\prime}\right) \cdot \frac{\vec{k}}{m} \frac{\beta}{m} \vec{p} \cdot \vec{\Phi}_{F h}(\vec{k}) f_{0}(p) \varphi_{h F}\left(\vec{k} ; \vec{p}^{\prime} ; t, t^{\prime}\right) \cdot \frac{\partial}{\partial \vec{p}^{\prime}} \\
& \quad+\frac{i \vec{k}}{m} \cdot \vec{p} \varphi_{n F}\left(\vec{k} ; \vec{p}, \vec{p}^{\prime} ; t, t^{\prime}\right) \cdot \frac{\partial}{\partial \vec{p}}-\frac{\vec{k}}{m} \cdot \varphi_{\jmath n}\left(\vec{k} ; \vec{p}, \vec{p}^{\prime} ; t, t^{\prime}\right) \frac{\vec{k}}{m} \cdot \vec{p}^{\prime}-\frac{\partial}{\partial \vec{p}} \cdot \vec{p} \varphi_{F n}\left(\vec{k} ; \vec{p}, \vec{p}^{\prime} ; t, t^{\prime}\right) \frac{i \vec{k}}{m} \cdot \vec{p}^{\prime} \\
& \quad-\frac{\beta}{m} \vec{p} \cdot \vec{\Phi}_{F h}(\vec{k}) f_{0}(p) \varphi_{h n}\left(\vec{k} ; \vec{p}^{\prime} ; t, t^{\prime}\right) \frac{i \vec{k}}{m} \cdot \vec{p}^{\prime}+\frac{\vec{k}}{m} \cdot \vec{p} \varphi_{n n}^{(0)}\left(\vec{k} ; \vec{p}^{\prime} ; t, t^{\prime}\right) \frac{\vec{k}}{m} \cdot \vec{p}^{\prime} .
\end{aligned}
$$


It contains the following correlation functions

$$
\begin{array}{ll}
\varphi_{n n}^{(0)}\left(\vec{k} ; \vec{p}, \vec{p}^{\prime} ; t, t^{\prime}\right)=\left\langle\hat{n}_{\vec{k}}(\vec{p}) T_{0}\left(t, t^{\prime}\right) \hat{n}_{-\vec{k}}\left(\vec{p}^{\prime}\right)\right\rangle_{0}, & \varphi_{h n}\left(\vec{k} ; \vec{p}^{\prime} ; t, t^{\prime}\right)=\left\langle\hat{h}_{\vec{k}}^{i n t} T_{0}\left(t, t^{\prime}\right) \hat{n}_{-\vec{k}}\left(\vec{p}^{\prime}\right)\right\rangle_{0}, \\
\varphi_{h \jmath}\left(\vec{k} ; \vec{p}^{\prime} ; t, t^{\prime}\right)=\left\langle\hat{h}_{\vec{k}}^{i n t} T_{0}\left(t, t^{\prime}\right) \hat{\vec{\jmath}}_{-\vec{k}}\left(\vec{p}^{\prime}\right)\right\rangle_{0}, & \varphi_{h F}\left(\vec{k} ; \vec{p}^{\prime} ; t, t^{\prime}\right)=\left\langle\hat{h}_{\vec{k}}^{i n t} T_{0}\left(t, t^{\prime}\right) \hat{\vec{F}}_{-\vec{k}}\left(\vec{p}^{\prime}\right)\right\rangle_{0}, \quad(\mathrm{~A} \\
\varphi_{n \jmath}\left(\vec{k} ; \vec{p}, \vec{p}^{\prime} ; t, t^{\prime}\right)=\left\langle\hat{n}_{\vec{k}}(\vec{p}) T_{0}\left(t, t^{\prime}\right) \hat{\vec{\jmath}}_{-\vec{k}}\left(\vec{p}^{\prime}\right)\right\rangle_{0}, & \varphi_{n F}\left(\vec{k} ; \vec{p}, \vec{p}^{\prime} ; t, t^{\prime}\right)=\left\langle\hat{n}_{\vec{k}}(\vec{p}) T_{0}\left(t, t^{\prime}\right) \hat{\vec{F}}_{-\vec{k}}\left(\vec{p}^{\prime}\right)\right\rangle_{0}, \\
\varphi_{F n}\left(\vec{k} ; \vec{p}, \vec{p}^{\prime} ; t, t^{\prime}\right)=\left\langle\hat{\vec{F}}_{\vec{k}}(\vec{p}) T_{0}\left(t, t^{\prime}\right) \hat{n}_{-\vec{k}}\left(\vec{p}^{\prime}\right)\right\rangle_{0}, & \varphi_{h n}\left(\vec{k} ; \vec{p}^{\prime} ; t, t^{\prime}\right)=\left\langle\hat{h}_{\vec{k}}^{i n t} T_{0}\left(t, t^{\prime}\right) \hat{n}_{-\vec{k}}\left(\vec{p}^{\prime}\right)\right\rangle_{0},
\end{array}
$$

constructed on the basic set of dynamic variables $\hat{n}_{\vec{k}}(\vec{p}), \hat{h}_{\vec{k}}^{\text {int }}$ along with the Fouriercomponents of the momentum density $\hat{\vec{\jmath}}_{\vec{k}}(\vec{p})$ and the force $\vec{F}_{\vec{k}}(\vec{p})$ in momentum space. Moreover, $\hat{n}_{\vec{k}}(\vec{p}), \hat{\vec{j}}_{\vec{k}}(\vec{p})$ and $\vec{F}_{\vec{k}}(\vec{p})$ are connected by the equation of motion (3.1).

The correlation functions (A.2) enter into $\varphi_{n j}^{(2)}\left(\vec{k} ; \vec{p} ; t, t^{\prime}\right)$ from equation (3.8):

$$
\begin{gathered}
\varphi_{n \jmath}^{(2)}\left(\vec{k} ; \vec{p} ; t, t^{\prime}\right)=\frac{i \vec{k}}{m} \cdot \vec{p} \varphi_{n h}\left(\vec{k} ; \vec{p} ; t, t^{\prime}\right) \frac{\beta}{m n} \vec{\Phi}_{F h}(\vec{k})+\frac{\beta}{m} \vec{p} \cdot \vec{\Phi}_{F h}(\vec{k}) f_{0}(p) \varphi_{h h}\left(\vec{k} ; t, t^{\prime}\right) \frac{\beta}{m n} \vec{\Phi}_{F h}(\vec{k}) \\
-\frac{i \vec{k}}{m} \cdot \varphi_{\jmath h}\left(\vec{k} ; \vec{p} ; t, t^{\prime}\right) \frac{\beta}{m n} \vec{\Phi}_{F h}(\vec{k})+\frac{\partial}{\partial \vec{p}} \cdot \varphi_{F h}\left(\vec{k} ; \vec{p} ; t, t^{\prime}\right) \frac{\beta}{m n} \vec{\Phi}_{F h}(\vec{k}),
\end{gathered}
$$

where

$$
\begin{aligned}
\varphi_{j h}\left(\vec{k} ; \vec{p} ; t, t^{\prime}\right) & =\left\langle\hat{\vec{\jmath}}_{\vec{k}}(\vec{p}) T_{0}\left(t, t^{\prime}\right) \hat{h}_{-\vec{k}}^{i n t}\right\rangle_{0}, \varphi_{F h}\left(\vec{k} ; \vec{p} ; t, t^{\prime}\right)=\left\langle\vec{F}_{\vec{k}}(\vec{p}) T_{0}\left(t, t^{\prime}\right) \hat{h}_{-\vec{k}}^{i n t}\right\rangle_{0}, \\
\varphi_{h h}\left(\vec{k} ; t, t^{\prime}\right) & =\left\langle\hat{h}_{\vec{k}}^{i n t} T_{0}\left(t, t^{\prime}\right) \hat{h}_{-\vec{k}}^{i n t}\right\rangle_{0}
\end{aligned}
$$

the correlation functions of the Fourier-components of densities of the potential part of enthalpy, momentum $\hat{\vec{j}}_{\vec{k}}(\vec{p})$ and force $\vec{F}_{\vec{k}}(\vec{p})$ in impulse space.

The correlation functions entering the equation (3.14) have the following structure

$$
\begin{aligned}
& \bar{\varphi}_{j h}\left(\vec{k} ; \vec{p} ; t, t^{\prime}\right)=\left\langle\hat{\vec{j}}_{\vec{k}}(\vec{p}) T_{0}\left(t, t^{\prime}\right) \dot{\hat{h}}_{-\vec{k}}^{i n t}\right\rangle_{0} \Phi_{h h}^{-1}(\vec{k}), \\
& \bar{\varphi}_{F \dot{h}}\left(\vec{k} ; \vec{p} ; t, t^{\prime}\right)=\left\langle\vec{F}_{\vec{k}}(\vec{p}) T_{0}\left(t, t^{\prime}\right) \hat{\hat{h}}_{-\vec{k}}^{i n t}\right\rangle_{0} \Phi_{h h}^{-1}(\vec{k}), \\
& \bar{\varphi}_{h \dot{h}}\left(\vec{k} ; t, t^{\prime}\right)=\left\langle\hat{h}_{\vec{k}}^{i n t}(\vec{p}) T_{0}\left(t, t^{\prime}\right) \dot{\hat{h}}_{-\vec{k}}^{i n t}\right\rangle_{0} \Phi_{h h}^{-1}(\vec{k}), \\
& \bar{\varphi}_{n \dot{h}}\left(\vec{k} ; t, t^{\prime}\right)=-\left\langle\dot{\hat{n}}_{\vec{k}}(\vec{p}) T_{0}\left(t, t^{\prime}\right) \hat{h}_{-\vec{k}}^{i n t}\right\rangle_{0} \Phi_{h h}^{-1}(\vec{k})=\frac{i \vec{k}}{m} \cdot \bar{\varphi}_{\text {Jh }}\left(\vec{k} ; \vec{p} ; t, t^{\prime}\right)-\frac{\partial}{\partial \vec{p}} \cdot \bar{\varphi}_{F h}\left(\vec{k} ; \vec{p} ; t, t^{\prime}\right), \\
& \bar{\varphi}_{\text {Jh }}\left(\vec{k} ; \vec{p} ; t, t^{\prime}\right)=\varphi_{\text {Jh }}\left(\vec{k} ; \vec{p} ; t, t^{\prime}\right) \Phi_{h h}^{-1}(\vec{k}), \\
& \bar{\varphi}_{F h}\left(\vec{k} ; \vec{p} ; t, t^{\prime}\right)=\varphi_{F h}\left(\vec{k} ; \vec{p} ; t, t^{\prime}\right) \Phi_{h h}^{-1}(\vec{k})
\end{aligned}
$$


and they are the normalized time correlation functions.

[1] Physics of Simple Liquids, Temperley H.N.V., Rowlinson J.S., Rushbrooke G.S. (Eds.), NorthHolland, Amsterdam, 1968.

[2] Jhon M.S., Forster D., A kinetic theory of classical simple liquids, Phys. Rev. A, 1975, 12, 254

[3] Götze W., and Lücke M., Phys. Rev. A, 1975, 11, No. 6, 2173.

[4] Zippelius A., Götze W., Kinetic theory for the coherent scattering function of classical liquids, Phys. Rev. A, 1978, 17, No. 1, 414-423.

[5] Bogolubov N.N., On the stochastic processes in the dynamical systems, Particles \& Nuclei, 1978, vol. 9, No. 4, p. 501-579 (in Russian).

[6] Dynamics of Solids and Liquids by Neurton Scattering, Lovesey S.W., Schringer T. (Eds.), Berlin, Springer Verlag, 1977.

[7] Forster D., Hydrodynamic Fluctuations, Broken Symmetry, and Correlation Functions, W.A. Benjamin, Reading, Mass. (1975).

[8] March N.M., Tosi M.P., Atomic dynamic in liquids, The Gresham Press, Surrey, 1976

[9] Resibois P., de Leener M., Classical Kinetic Theory of Fluids, New York, Wiley, 1977.

[10] Boon J.P., Yip S., Molecular Hydrodynamics, Dover, New York, 1980.

[11] Zubarev D.N., Morozov V.G., Formulation of boundary conditions for the BBGKY hierarchy with allowance for local conservation laws, Theor. Math. Phys., 1984, 60, 814;

[12] Zubarev D.N., Morozov V.G., Omelyan I.P., Tokarchuk M.V., Kinetic equations for dense gases and liquids, Theor. Math. Phys., 1991, 87, 412;

[13] Klimontovich Yu.L., On the need for and the possibility of a unified description of kinetic and hydrodynamic processes, Theor. Math. Phys., 1992, 92, 909;

[14] Shurygin V.Yu, Yulmetyev R.M., Influence of non-Markovian effects in thermal motion of particles on intensity of non-coherent scattering of slow neutrons in liquids, Teor. Mat. Fiz., 1990, vol. 83, No. 2, p. 222-235 (in Russian).

[15] Shurygin V.Yu, Yulmetyev R.M., Calculation of the dynamic structure factor for liquids by the reduced description technique, Zhurn. Exp. Teor. Fiz., 1989, vol. 96, No. 3(9), p. 938-947 (in Russian). 
[16] Shurygin V.Yu, Yulmetyev R.M., Space dispersion of structure relaxation in simple liquids, Zhurn. Exp. Teor. Fiz., 1991, vol. 99, No. 1, p. 144-154 (in Russian).

[17] Shurygin V.Yu, Yulmetyev R.M., On spectre of non-Markovianity of relaxation processes in liquids, Zhurn. Exp. Teor. Fiz., 1992, vol. 102, No. 3(9), p. 852-862 (in Russian).

[18] Shurygin V.Yu, Yulmetyev R.M., Role of asymmetric time correlation functions in kinetics of relaxation processes in liquids, Ukr. J. Phys., 1990, vol. 35, No. 5, p. 693-695 (in Russian).

[19] Tokarchuk M.V., On the statistical theory of a nonequilibrium plasma in its electromagnetic self-field, Theor. Math. Phys., 1993, 97, 1126;

[20] Zubarev D.N., Morozov V.G., Omelyan I.P., Tokarchuk M.V., Unification of the kinetic and hydrodynamic approaches in the theory of dense gases and liquids, Theor. Math. Phys., 1993, 96, 997;

[21] Tokarchuk M.V., Omelyan I.P., Kobryn A.E., A consistent description of kinetics and hydrodynamics of systems of interacting particles by means of the nonequilibrium statistical operator method, Condens. Matter Phys., 1998, 1, 687.

[22] Zubarev D.N., Morozov V.G., Röpke G., Statistical Mechanics of Nonequilibrium Processes. Vol. 1: Basic Concepts, Kinetic Theory, Akademie Verlag, Berlin, 1996.

[23] Luzzi R., Vasconcellos A.R., Ramos J.G., The theory of irreversible processes: Foundations of a non-equilibrium statistical ensemble formalism, Rivista del Nuovo cimento, 2006, vol. 29, No 2, p.1-82.

[24] Vasconcellos A.R., Ramos J.G., Luzzi R., Ensemble Formalism and an Associated Irreversible Statistical Thermodynamics, Bras. J. Phys., 2005, vol.35, No 3A, p.689-717.

[25] Silva C.A.B., Vasconcellos A.R., Ramos J.G., Luzzi R., Generalized Kinetic Equation for Far-from-Equilibrium Many-Body Systems, J. Stat. Phys., 2011, vol. 143, p. 1020-1034.

[26] Silva C.A.B., Ramos J.G., Vasconcellos A.R., Luzzi R., Nonlinear higher-order hydrodynamics: Unification of kinetic and hydrodynamic within a nonequilibrium statistical ensemble formalism. arXiv.: 1210.7280v1[physics. flu-dyn], 2012, 29p.

[27] Tsytovich V.N., de Angelis U., Kinetic theory of dusty plasmas. V. The hydrodynamic equiations, Phys. Plasmas, 2004, vol. 11, p.496-506.

[28] Tokarchuk M.V., Markiv B.B., Collected Physical Papers, Shevchenko Scientific Society, 2008, 7, 100 (in Ukrainian).

[29] van Well A.A., Verkerk P., and de Graaf L.A., Suck J.-B., Copley J.R.D., Phys.Rev. A, 1985, 
vol. 31, No 5, p. 3391-3414.

[30] Bulavin L.A., Neutron diagnostics of liquid state matter, Institute of safety of nuclear power plants, Chornobyl, 2012., 532 p. (in Ukrainian).

[31] De Schepper I.M., Cohen E.G.D., Very-short-wavelength collective modes in fluids, J. Stat. Phys., 1982, 27, 223;

[32] Zubarev D.N., Nonequilibrium Statistical Thermodynamics, Consultant Bureau, New-York, 1974 .

[33] Markiv B.B., Omelyan I.P, Tokarchuk M.V., On the problem of a consistent description of kinetic and hydrodynamic processes in dense gases and liquids, Condens. Matter Phys., 2010, 13, 23005;

[34] Akcasu A.Z., Duderstadt J.J., Derivation of kinetic equations from the generalized Langevin equation, Phys. Rev., 1969, 188, 479.

[35] Forster D., Martin P.C., Kinetic theory of a weakly coupled fluid, Phys. Rev. A, 1970, 2, 1575.

[36] Furtado P.M., Mazenko G.F., Yip S., Hard-sphere kinetic-theory analysis of classical, simple liquids, Phys. Rev. A, 1975, 12, 1653;

[37] Mazenko G.F., Microscopic Method for Calculating Memory Functions in Transport Theory, Phys. Rev. A, 1971, 3, 2121;

[38] Mazenko G.F., Properties of the Low-Density Memory Function, Phys. Rev. A, 1972, 5, 2545;

[39] Mazenko G.F., Tomas Y.S., Yip S., Thermal Fluctuations in a Hard-Sphere Gas, Phys. Rev. A, 1972, 5, 1981;

[40] Mazenko G.F., Fully Renormalized Kinetic Theory. I. Self-Diffusion, Phys. Rev. A, 1973, 7, 209.

[41] Mazenko G.F., Fully Renormalized Kinetic Theory. II. Velocity Autocorrelation, Phys. Rev. A, 1973, 7, 222 .

[42] Mazenko G.F., Fully renormalized kinetic theory. III. Density fluctuations, Phys. Rev. A, $1974, \mathbf{9}, 360$.

[43] Forster D., Properties of the kinetic memory function in classical fluids, Phys. Rev. A, 1974, 9, 943 .

[44] Boley C.D., Kinetic Theory of a Dense Gas: Triple-Collision Memory Function, Desai R.C. Phys. Rev. A, 1973, 7, 1700.

[45] Sjodin S., Kinetic model for classical liquids, Sjolander A. Phys. Rev. A, 1978, 18, 1723. 
[46] Balucani U., Zoppi M. Dynamics of the Liquid State. Clarendon Press, Oxford, 1994.

[47] Mryglod I.M., Omelyan I.P., Tokarchuk M.V., Generalized collective modes for the LennardJones fluid, Mol. Phys., 1995, 84, 235.

[48] Mryglod I.M., Tokarchuk M.V., On statistical hydrodynamics of simple lquids, Prob. At. Sci. Technol., 1992, 3, 134.

[49] De Schepper I.M., Cohen E.G.D., Collective modes in fluids and neutron scattering, Phys. Rev. A, 1980, 22, 287;

[50] Chapman S., Cowling T.G., The Mathematical Theory of Non Uniform Gases, Cambrige University Press, London, 1970.

[51] Markiv B.B., Omelyan I.P., Tokarchuk M.V., On the problem of a consistent description of kinetic and hydrodynamic processes in dense gases and liquids: Collective excitations spectrum, Condens. Matter Phys., 2012, 15, 14001. 